\title{
Multifeed EBG Dual-Band Antenna for Spatial Mission
}

\author{
A. Kanso, R. Chantalat, U. Naeem, H. Chreim, M. Thevenot, S. Bila, and T. Monediere
}

XLIM UMR CNRS No. 6615, University of Limoges, 123, avenue Albert Thomas, 87060 Limoges, France

Correspondence should be addressed to A. Kanso, ahmad.kanso@xlim.fr

Received 26 May 2011; Accepted 27 August 2011

Academic Editor: Z. Chen

Copyright (C) 2011 A. Kanso et al. This is an open access article distributed under the Creative Commons Attribution License, which permits unrestricted use, distribution, and reproduction in any medium, provided the original work is properly cited.

\begin{abstract}
We present the design of a multibeam reflector antenna fed by a multifeed dual-band electromagnetic band gap (EBG) antenna to achieve a high-gain multispot coverage for space applications. First, we design a dual-band EBG antenna in monofeed configuration. This antenna is composed of double-layer frequency selective surfaces (FSSs) arranged in the longitudinal direction and a square horn as a feed. Then, the same antenna in multifeed configuration is studied, and the results are compared to those obtained in monofeed configuration in order to emphasize the problem of coupling, generally encountered in multifeed configuration. Consequently, filters are used in order to reduce the parasitic interferences and obtain good radiation characteristics. As shown in this paper, the same EBG phase center is obtained in both frequency bands. Finally, we have studied the whole system composed of the offset reflector and the multifeed EBG dual-band antenna. An edge of coverage (EOC) gain higher than $42 \mathrm{dBi}$ and sidelobes levels lower than $-18 \mathrm{dBi}$ are obtained over all spots.
\end{abstract}

\section{Introduction}

Recently, the interest in using multibeam antennas to achieve communication links between satellite and earth has grown due to their ability to efficiently cover large regions. These antennas typically provide a contiguous coverage of a geographic region using high-gain multispots beams for both transmit and receive links; see Figure 1.

To meet the spatial coverage requirements, parabolic reflector with a cluster of feeds on the focal plane attract considerable attention as a potentially very cost-effective solution for multibeams generation. The goals of multibeam antenna are to maximize the EOC gain over the coverage region and minimize the interferences between each beam. In order to satisfy operators requirements in terms of channel's capacity and high gain, the use of small beams $\left(0.65^{\circ}\right.$ of diameter, spacing of $0.56^{\circ}$ ) is mandatory. In order to support both TX and RX links, the spatial application requires two independent bands, one for TX in Ka-band and one for RX in K-band. These objectives can be achieved by several systems.

The first system adopts, for each transmit and receive frequency band, one feed per beam concept, where adjacent beams are generated from different reflectors. This conventional antenna system requires four TX reflectors and four RX reflectors associated to focal arrays, to generate multibeams [1]. This system is an improved solution and the most often used with actual antenna generation. However, the nature of the mechanical design calls for a cumbersome approach.

Another procedure to generate a multibeam spot using a single reflector for each frequency bands is the focal array fed reflector [2]. This system utilizes a cluster of horns instead of a single one to generate each beam, some of these horns being used for several beams.

The third system uses common reflectors to generate both TX and RX bands and a separate cluster of feed horns for each band. A dichroic frequency-selective subreflector aligns the primary patterns of the two bands. For the low frequency band, the antenna works as a primary focus fed parabolic reflector; for the high-frequency band, it works as a Cassegrain antenna [3]. In order to generate multiple spots, the previous system requires four common reflectors.

Another approach uses one reflector fed by a cluster of multifrequency horns for both downlink and uplink [4]. In order to generate multiple spots coverage with this system, the number of reflector is divided by two compared to conventional antenna.

All these kinds of systems are good candidates for multibeams spatial applications but suffer from different 


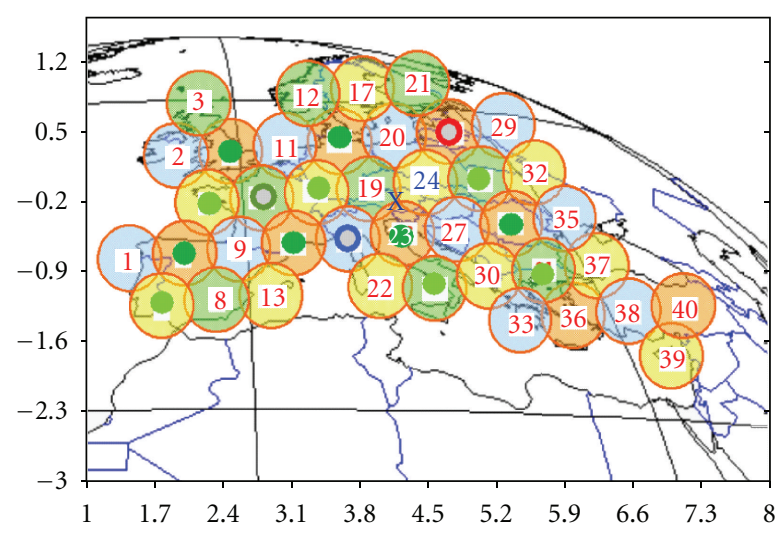

(a)

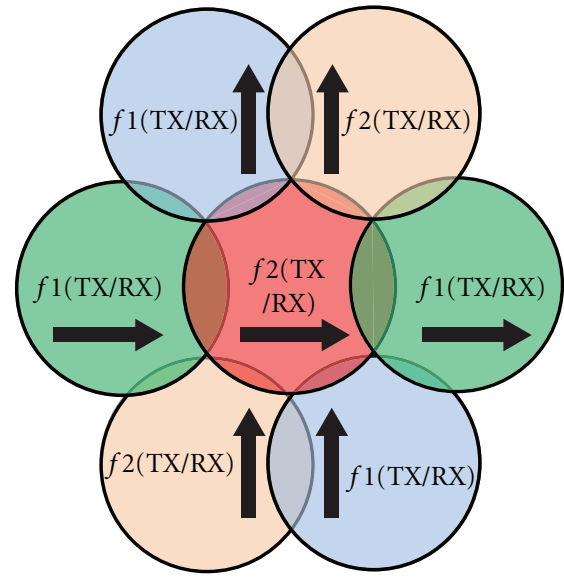

(b)

FIGURE 1: European multibeam coverage with $4 \mathrm{x}$ reuse scheme.

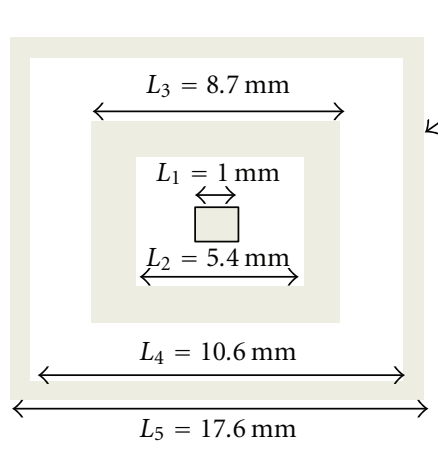

FSS

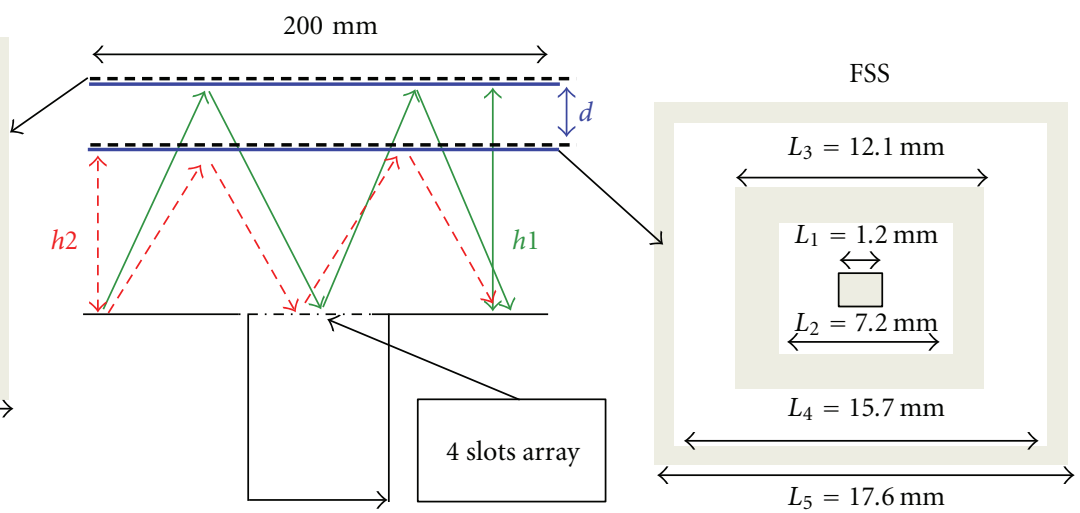

Wave guide

FIgURE 2: EBG dual-band antenna fed by a rectangular waveguide.

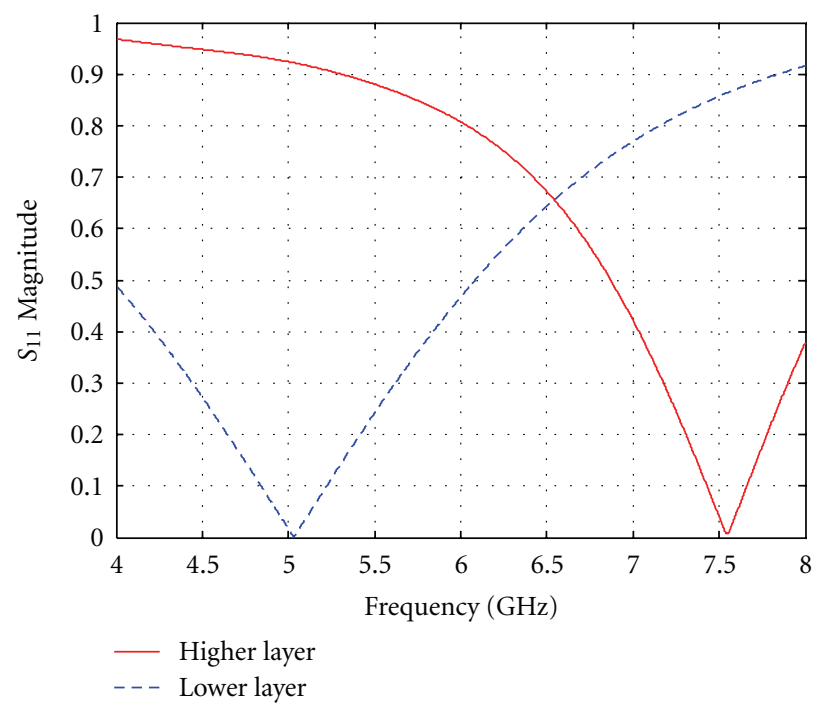

(a)

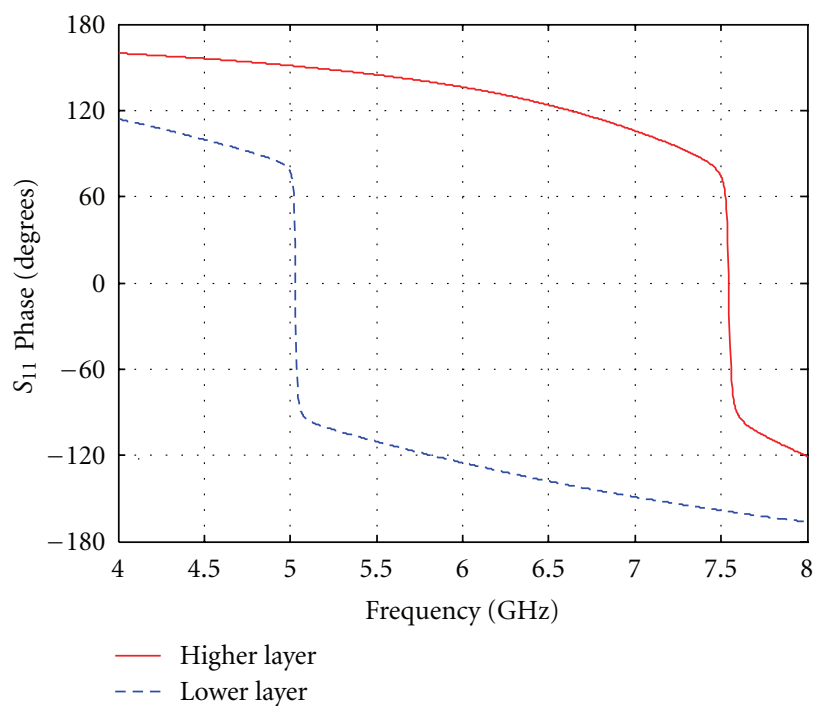

(b)

Figure 3: Reflection coefficient (a) magnitude and (b) phase of each FSS layer. 


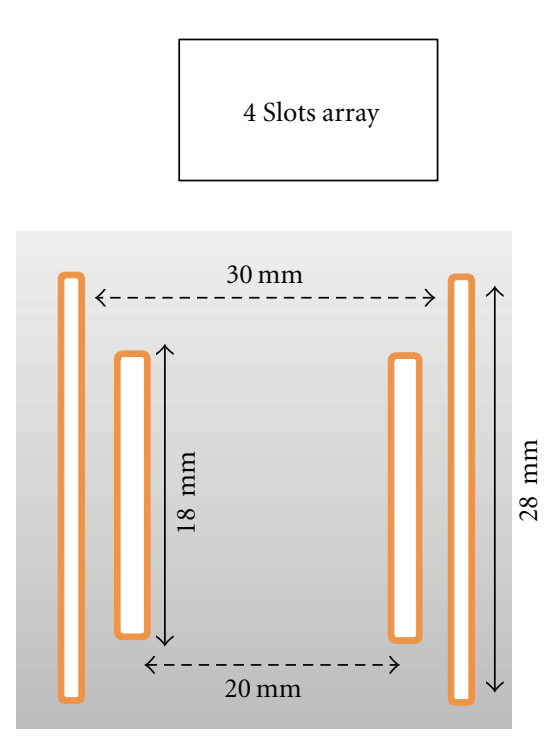

(a)

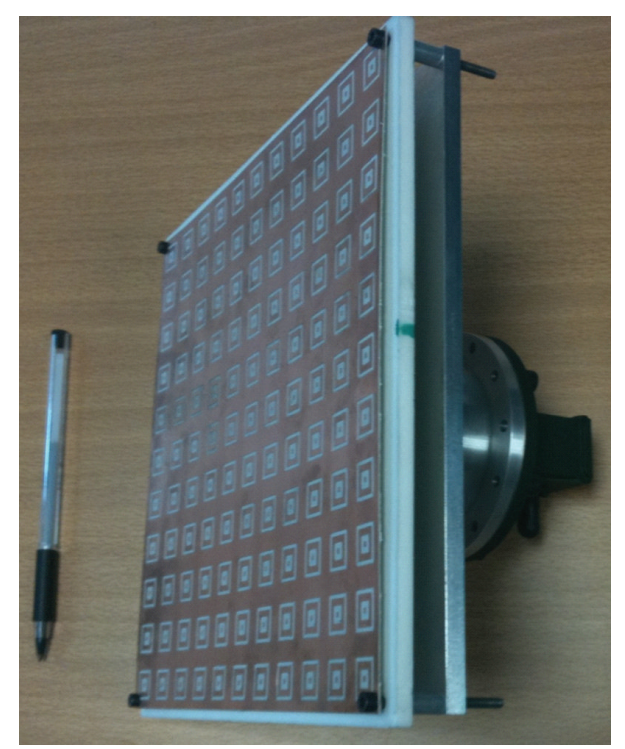

(b)

FIgURE 4: Photography of a 4 slots array $\lambda / 2$ (a) and the manufactured EBG dual-band antenna (b).

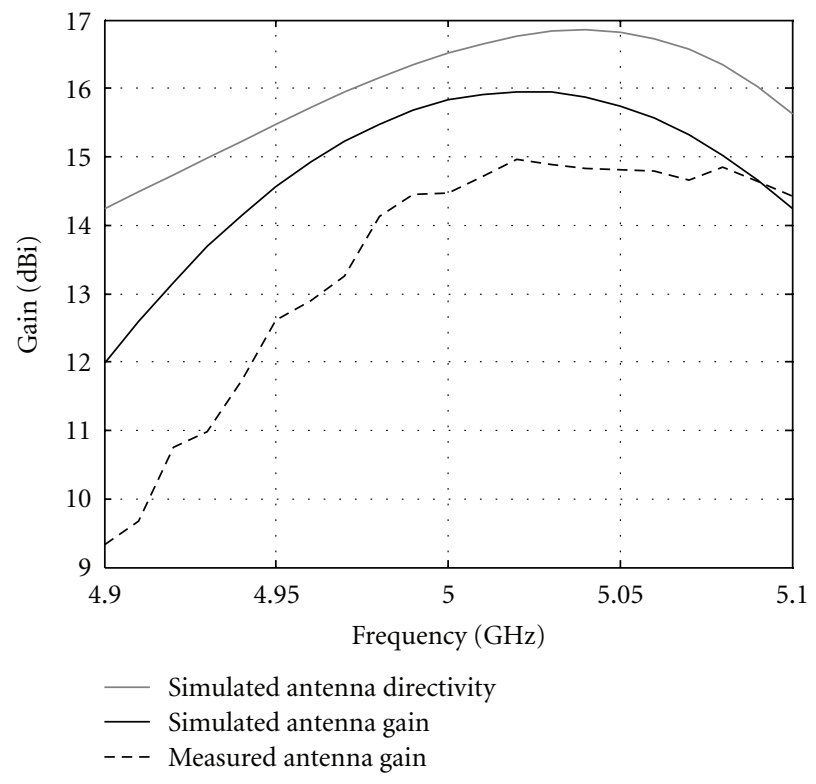

(a)

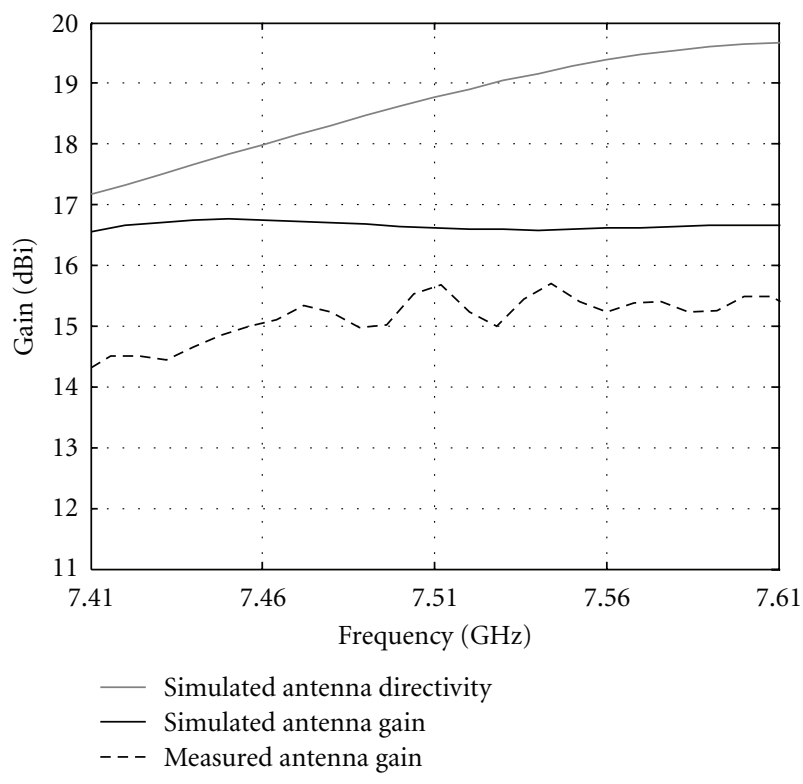

(b)

FIGURE 5: Simulated and measured gain versus frequency at $f_{L}(\mathrm{a})$ and $f_{H}(\mathrm{~b})$.

implementation difficulties. These approaches have the disadvantage of accommodating large number of reflectors on the spacecraft as well as their expensive cost.

In order to minimize the number of (TX/RX) reflector antennas, a multifeed EBG focal structure is proposed. The EBG antennas were developed several years ago and have been used in different applications [5]. Several studies have shown that it is possible to produce overlapped apertures by using multifeed EBG antenna [6, 7]. Moreover, such an antenna could be modified and designed to exhibit directive patterns for multiple distinct frequencies, and hence, it becomes a dual-band EBG antenna [8]. In the latter work, a design of a dual-band monofeed EBG resonator antenna which feeds a reflector for spatial applications (TX/RX) is proposed. This application requires two independent far-off bands (the RX band at $20 \mathrm{GHz}\left(f_{L}\right)$ and the TX band at $30 \mathrm{GHz}\left(f_{H}=1.5 * f_{L}\right)$. Moreover, the same phase center and the same radiation patterns for both frequency bands are needed.

In this paper, a dual-band multifeed EBG focal structure is described at $5 \mathrm{GHz}\left(f_{L}\right)$ and at $7.5 \mathrm{GHz}\left(f_{H}=1.5 *\right.$ $f_{L}$ ) for the sake of low manufacturing cost. The goal is 


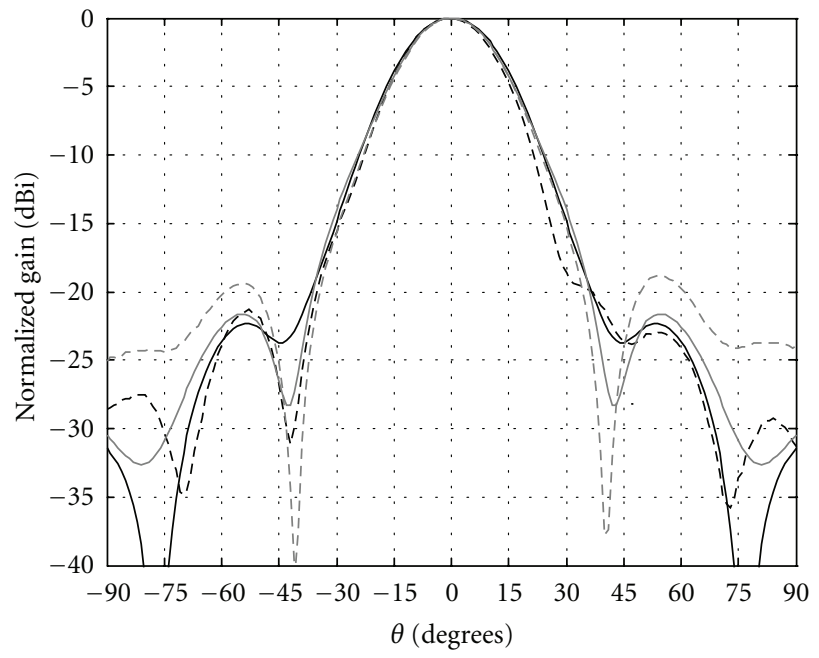

$\begin{array}{llll}- & \text { E plane: simulation } & - & \text { H plane: simulation } \\ --- & \text { E plane: measurement } \quad--- & \text { H plane: measurement }\end{array}$

(a)

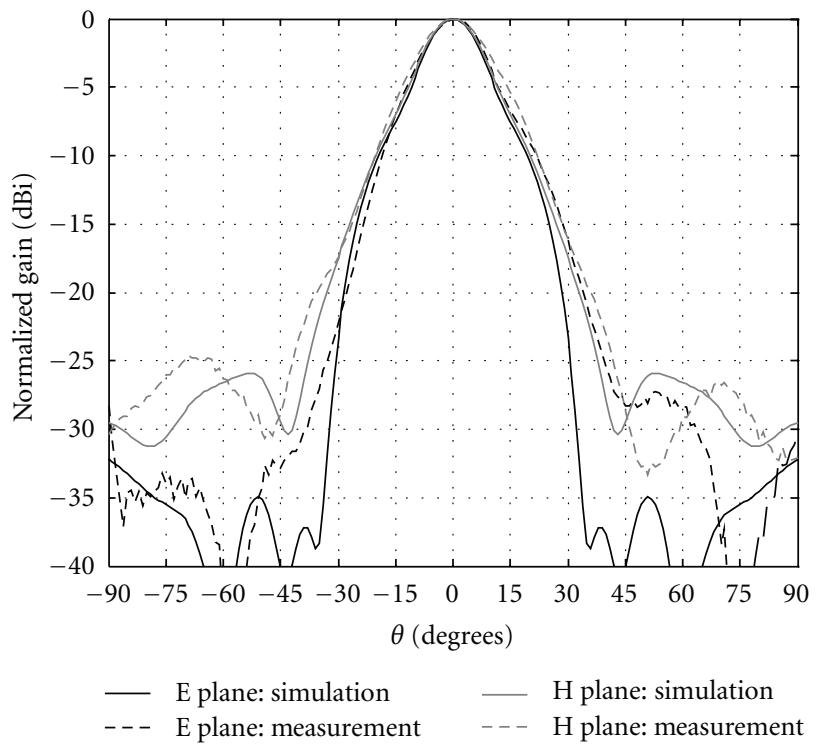

(b)

Figure 6: Normalized patterns at $5 \mathrm{GHz}$ (a) and $7.5 \mathrm{GHz}$ (b) in the $\mathrm{E}$ and $\mathrm{H}$ planes.

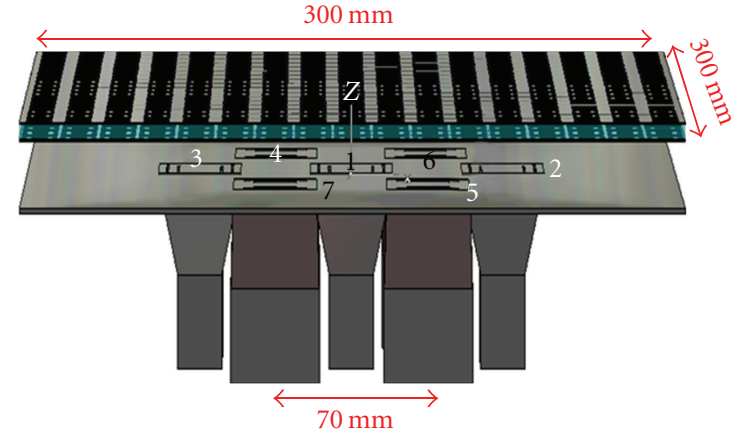

(a)

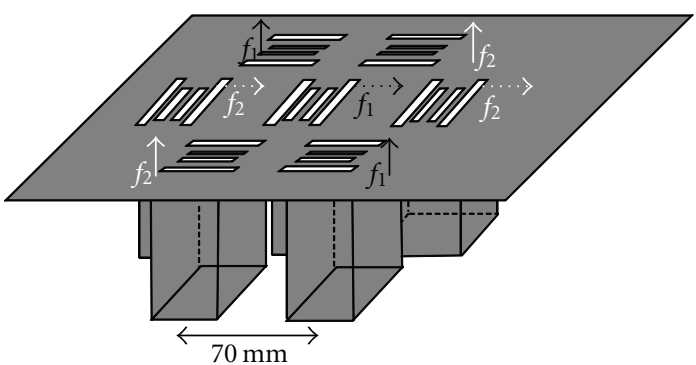

(b)

Figure 7: Multifeed EBG dual-band antenna (a) excited by 7 horns (b).

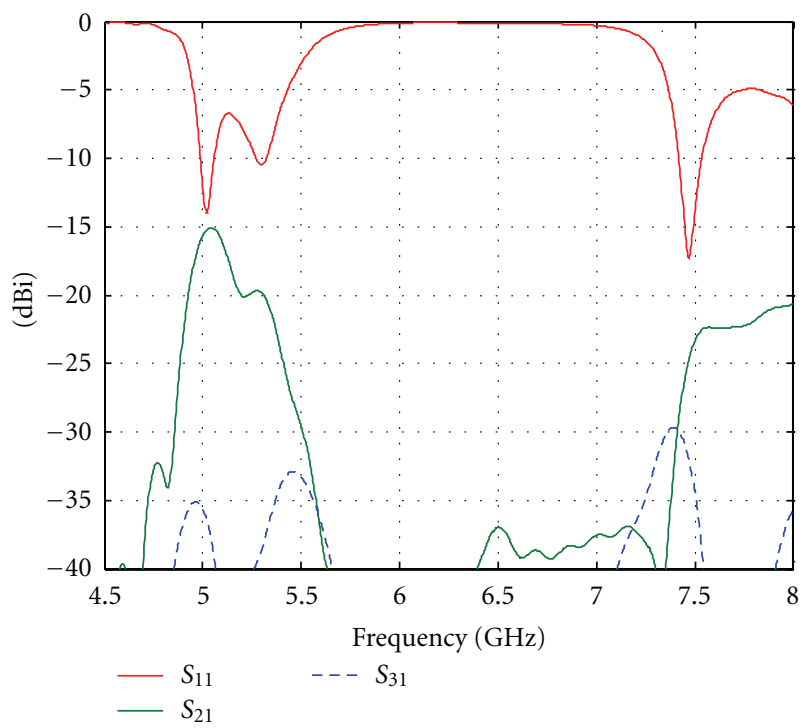

FIgURE 8: S-parameters of the multifeed EBG antenna when port 1 is excited. 


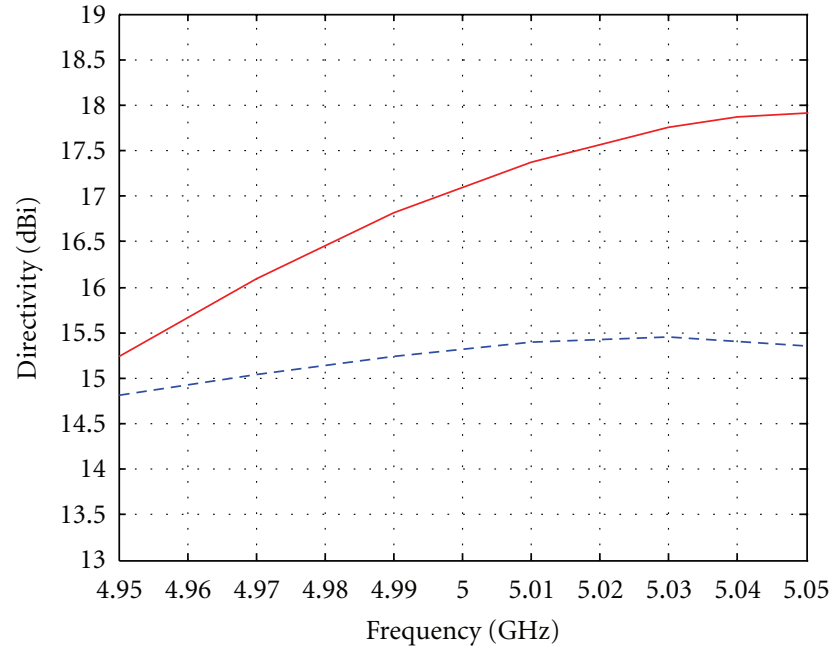

- Monofeed

-- - Multifeed

(a)

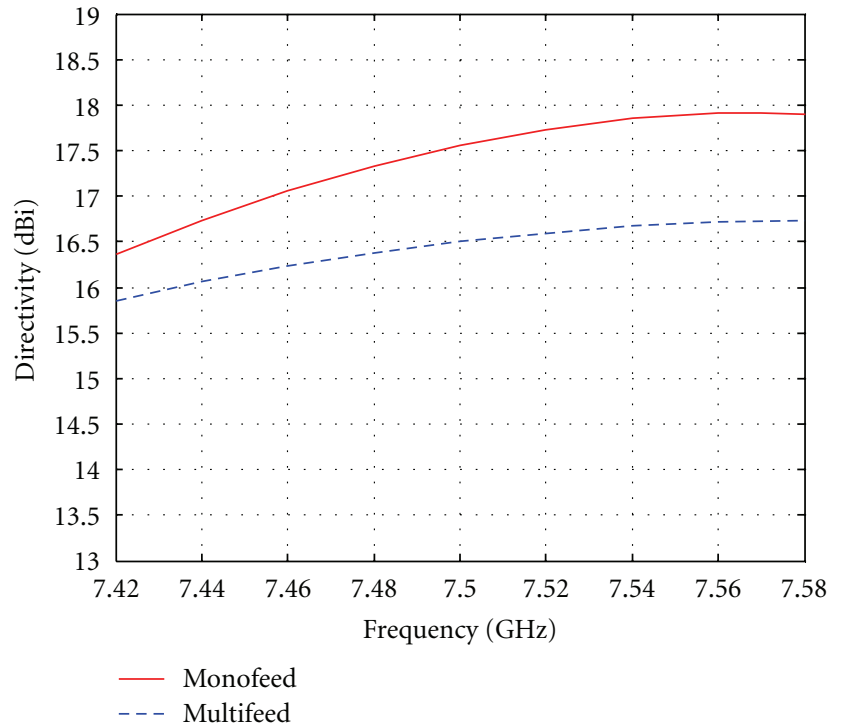

(b)

Figure 9: EBG dual-band antenna directivity at $f_{L}$ (a) and $f_{H}(\mathrm{~b})$.

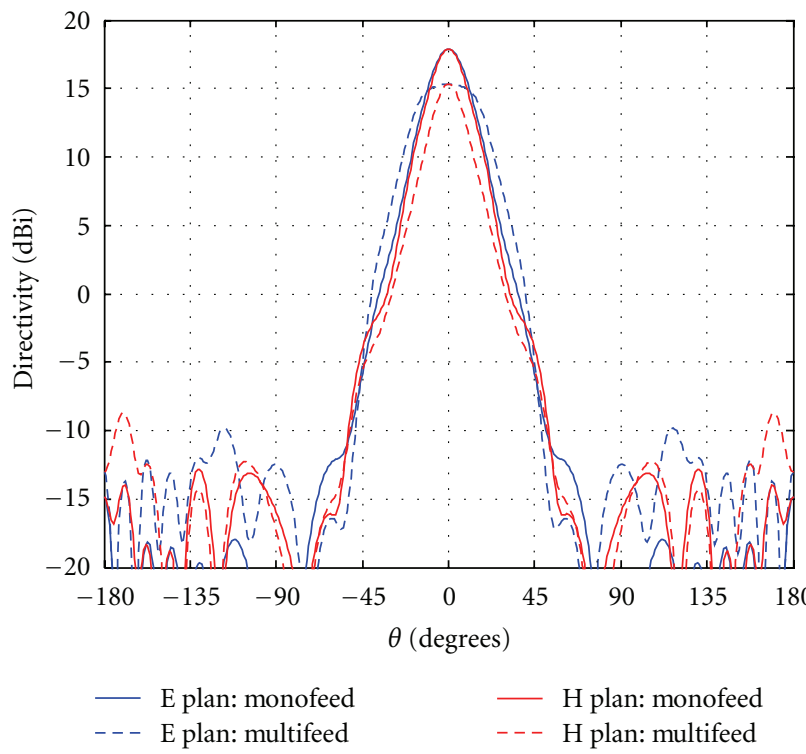

(a)

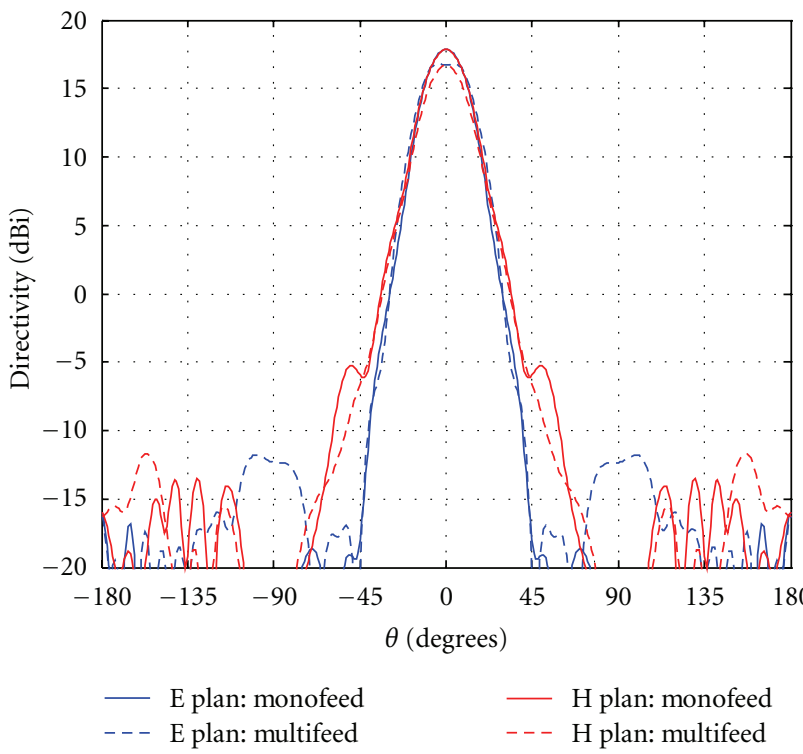

(b)

Figure 10: Radiation patterns at $5.05 \mathrm{GHz}$ (a) and $7.58 \mathrm{GHz}$ (b) in the $\mathrm{E}$ and $\mathrm{H}$ planes.

to reduce the parasitic effect between the EBG antenna ports (coupling). The latter problem can be resolved by connecting appropriate filters to the antenna ports which isolate frequency channels and help restoring the original undisturbed directivity.

This paper is organized in three parts. In the first part, we present the objective of the mission. In the second part, after presenting the design and the performances of the dual-band monofeed EBG antenna, we describe the one in multifeed configuration and then the filter dual-band devices will be inserted upstream the EBG antenna in order to isolate the frequency channels and reconstruct the radiation patterns.
Finally, the coverage performances after illuminating the reflector by this antenna will be given.

\section{Coverage Specifications and Design Requirement}

The objective is to provide a European multibeam coverage at $5 \mathrm{GHz}$ and $7.5 \mathrm{GHz}$ with 40 interlaced spots of $0.65^{\circ}$ from a geostationary satellite. This coverage is dedicated to high data rate telecommunications and uses a $4 \mathrm{x}$ reuse scheme $(2 \times 250 \mathrm{MHz}$ frequency channels and 2 linear polarizations), as shown in Figure 1. Originally, the two frequency bands 


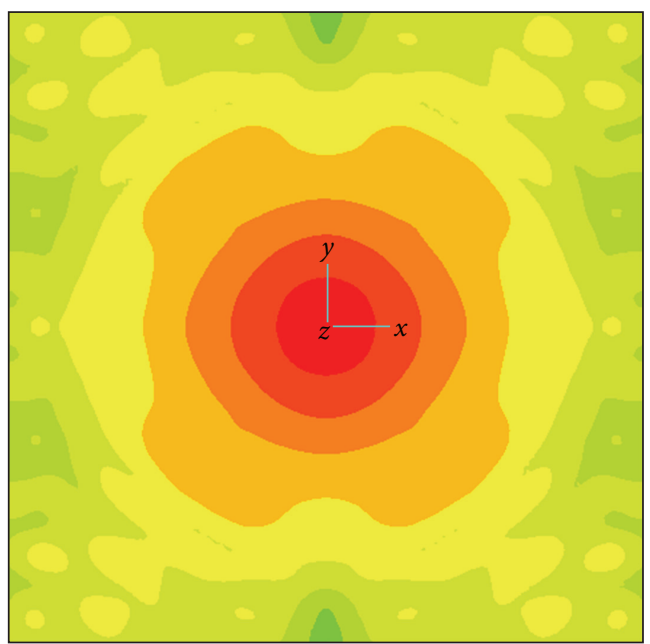

(a)

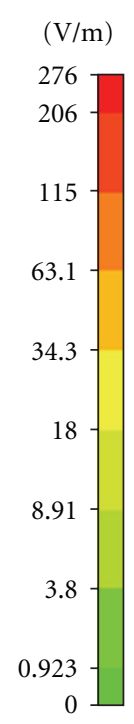

FIGURE 11: E-field distribution above antenna at $5.05 \mathrm{GHz}$ with one horn (a) and with 7 horns (b).

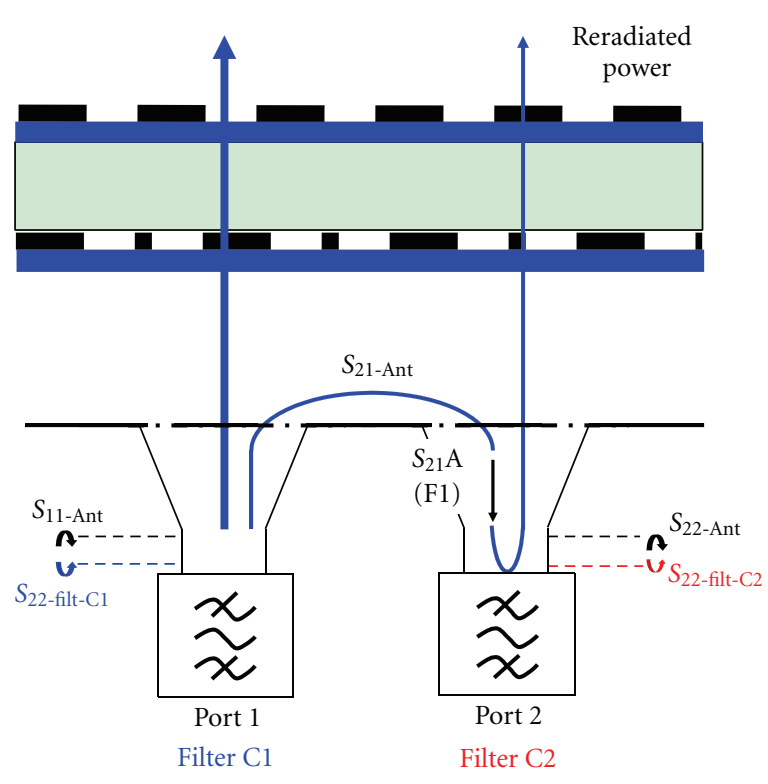

FIGURE 12: Multifeed EBG antenna loaded by filters.

are divided into two $250 \mathrm{MHz}$ subbands between 19.5$20 \mathrm{GHz}$ and $29.5-30 \mathrm{GHz}$ avoiding parasitic interferences between adjacent beams. As we said before, for the sake of low manufacturing cost and fabrication simplicity, we will conduct our study in lower frequency bands: $5 \mathrm{GHz}$ $(\mathrm{RX})$ and $7.5 \mathrm{GHz}\left(1.5 * f_{0}\right)$. The lower frequency band is divided into two $50 \mathrm{MHz}$ subbands between $4.95-5.05 \mathrm{GHz}$ (RX) and the higher frequency band is divided into two $75 \mathrm{MHz}$ subbands between $7.42-7.58 \mathrm{GHz}$. The coverage performance requirements are the following.

(i) The same reflector antenna illumination for both frequency bands. In this case, the dual-band EBG antenna must presents the same radiation patterns for both frequency bands.

(ii) As the EBG focal feed phase center must be placed at the reflector focus, the phase center location must be identical for both frequency bands. The phase center is considered as the reference point that allows a minimum variation of the far field radiation phase within a defined solid angle $\theta_{S}$.

(iii) A minimum coupling between ports is needed for both frequency bands.

(iv) The EOC gain, defined by the level at the triple beam crossover, will be higher than $42 \mathrm{dBi}$.

\section{EBG Antenna Design}

The first step is the design of a monofeed dual-band EBG antenna fed by a horn. In fact, a classical EBG antenna fed by patch or by a dipole suffers from the well-known problem of low radiation bandwidth and high sidelobes. On the other hand, the use of a horn as an EBG antenna feed increase the directivity and decrease the sidelobes of the EBG antenna without decreasing the radiation bandwidth. This result leads to a better reflector illumination law [5].

The second step is the design of a multifeed dual-band EBG antenna. The difficulty is to reduce the high coupling between ports which disturbs the radiation patterns. In order to reconstruct the radiation patterns, dual-band filters must be connected to each antenna ports.

\subsection{EBG Dual-Band Antenna in Monofeed Configuration.} The EBG dual-band antenna for spatial mission operating at $5 \mathrm{GHz}\left(f_{L}\right)$ and $7.5 \mathrm{GHz}\left(f_{H}\right)$ is presented in Figure 2.

This antenna is composed of two pass-band FSS separated by a distance $d$. The layers were printed on a dielectric slab whose thickness is equal to $0.5 \mathrm{~mm}$ and the 


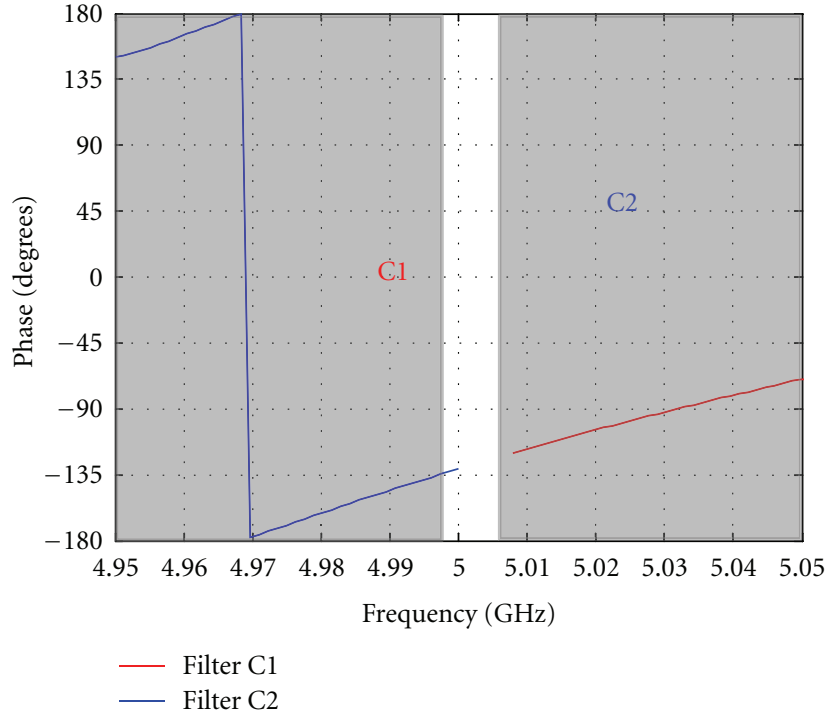

(a)

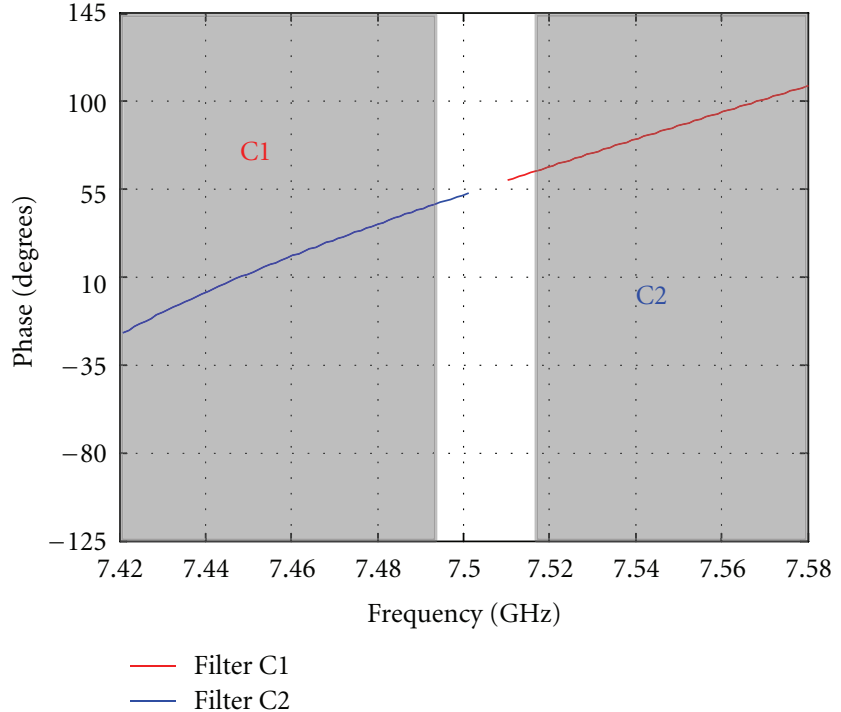

(b)

FIGURE 13: S22 ideal phase profile for each filter outside its operating band at $f_{L}$ (a) and $f_{H}(\mathrm{~b})$.

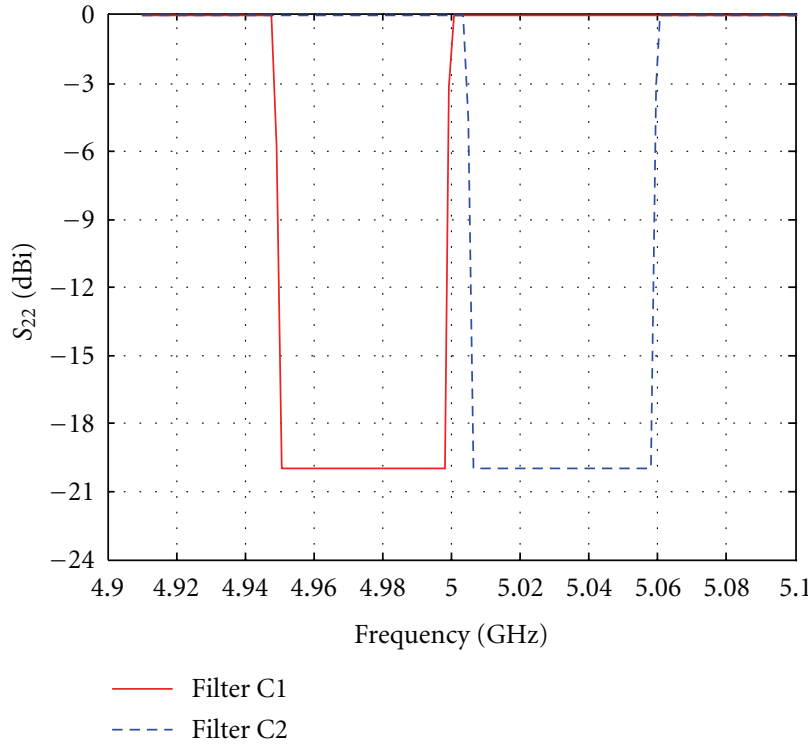

(a)

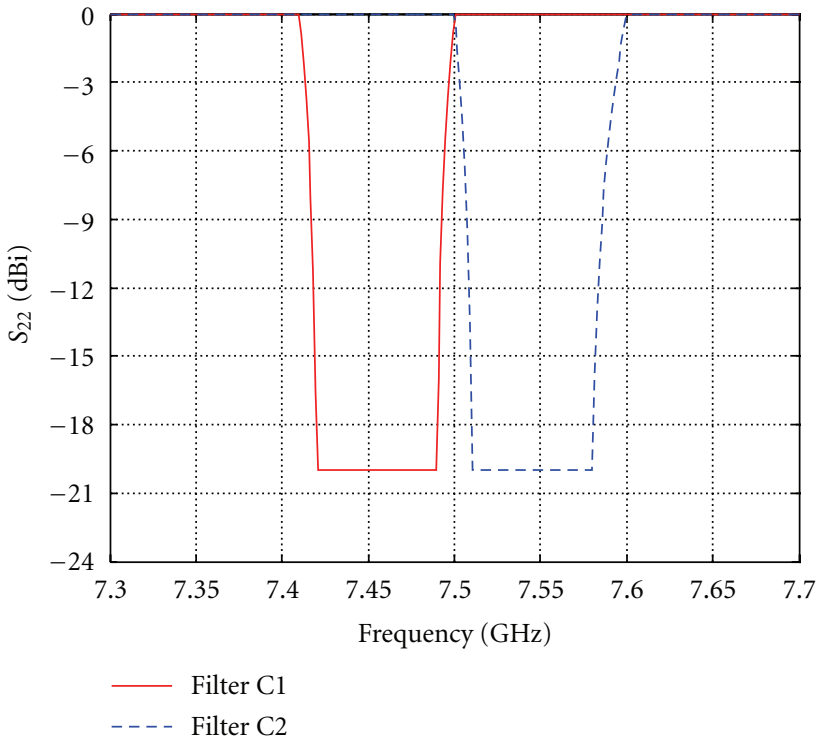

(b)

FIGURE 14: S22 magnitude of each filter at $f_{L}(\mathrm{a})$ and $f_{H}(\mathrm{~b})$.

dielectric permittivity is 2.94 . The bottom layer is chosen to be transparent at the lowest band and to achieve a reflection coefficient magnitude about 0.87 at the highest one. Likewise, the top layer is chosen to be transparent at the highest band and to achieve a reflection coefficient magnitude about 0.9 at the lowest one (Figure 3(a)).

As shown in Figure 2, the highest layer forms with the ground plane a first cavity with a height of $h 1$ whose resonance frequency is located inside the lowest band. The highest operating frequency is provided by the resonance of the second cavity that is formed between the ground plane and the FSS located at $h 2$. The cavity heights $h 1$ and $h 2$ can be calculated from (1) and their values are equal to $27.5 \mathrm{~mm}$ and $21.1 \mathrm{~mm}$, respectively,

$$
h=\frac{\lambda}{2}\left(\frac{\varphi+\pi}{2 \pi}\right),
$$

where $\varphi$ is the phase of the EBG mirror reflection coefficient given in Figure $3(\mathrm{~b})$ and $\lambda$ is the wavelength.

In order to match the EBG antenna over both frequency bands, two pairs of half wavelength slots are used (Figure 4(a)). Each pair of slots is excited in phase by the fundamental mode of a square horn $(40 * 40 \mathrm{~mm})$ that is fed by a standard waveguide. The photography of the 


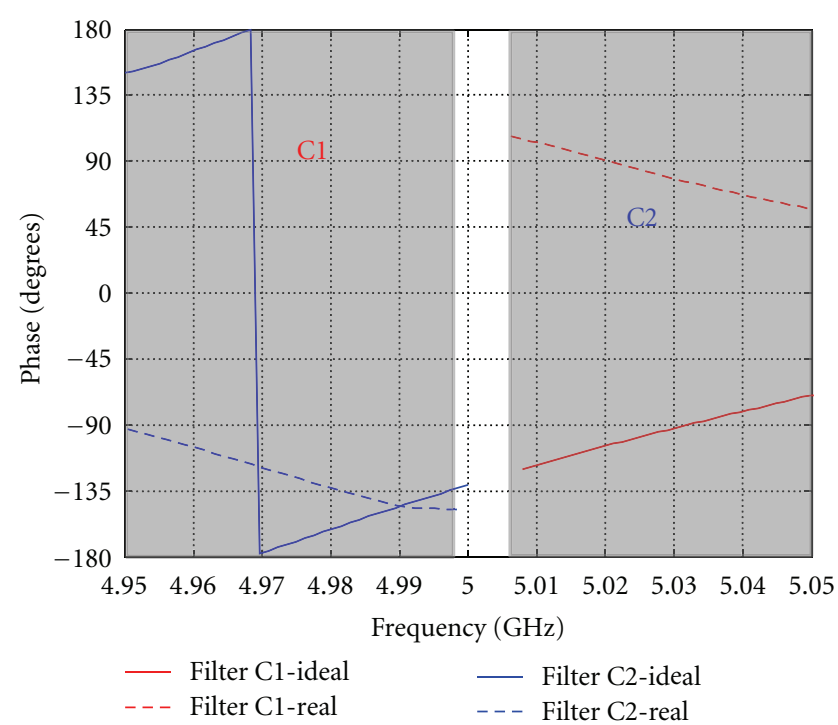

(a)

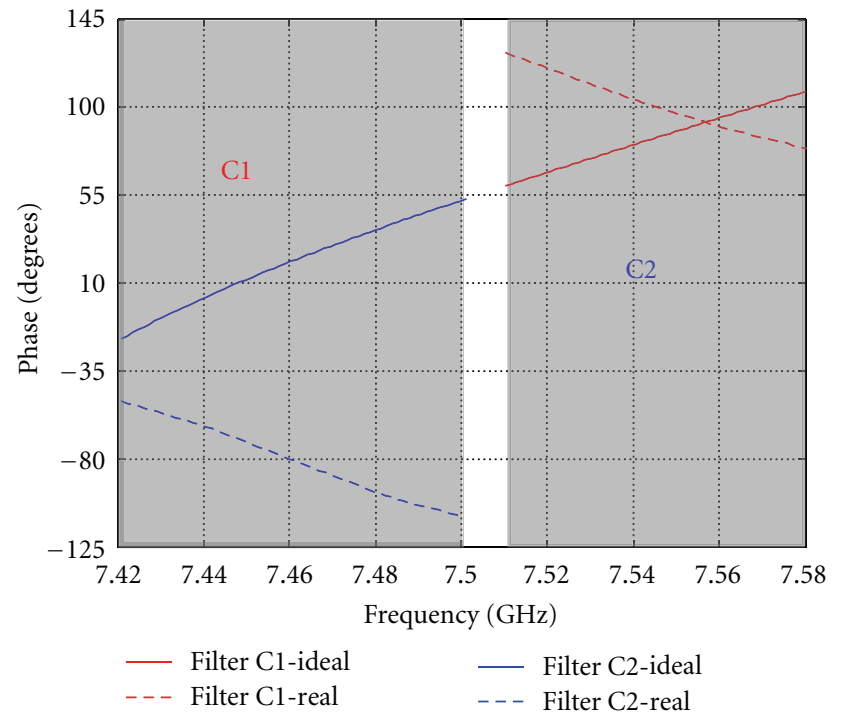

(b)

FIgURE 15: Out-of-band S22 phases profile of real filters, compared to the ones of ideal filters at $f_{L}(\mathrm{a})$ and $f_{H}(\mathrm{~b})$.

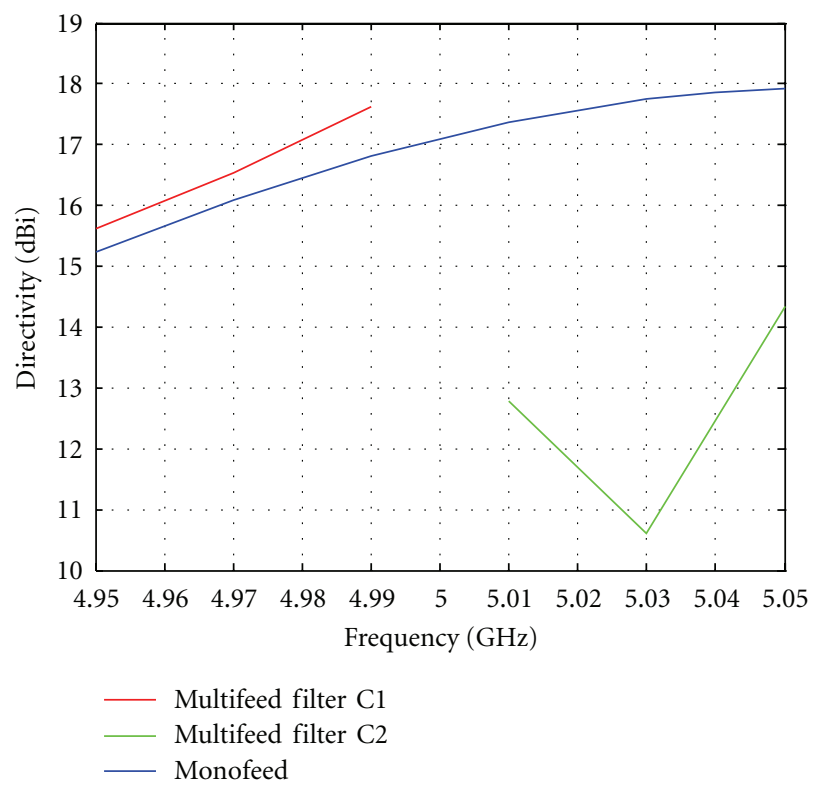

(a)

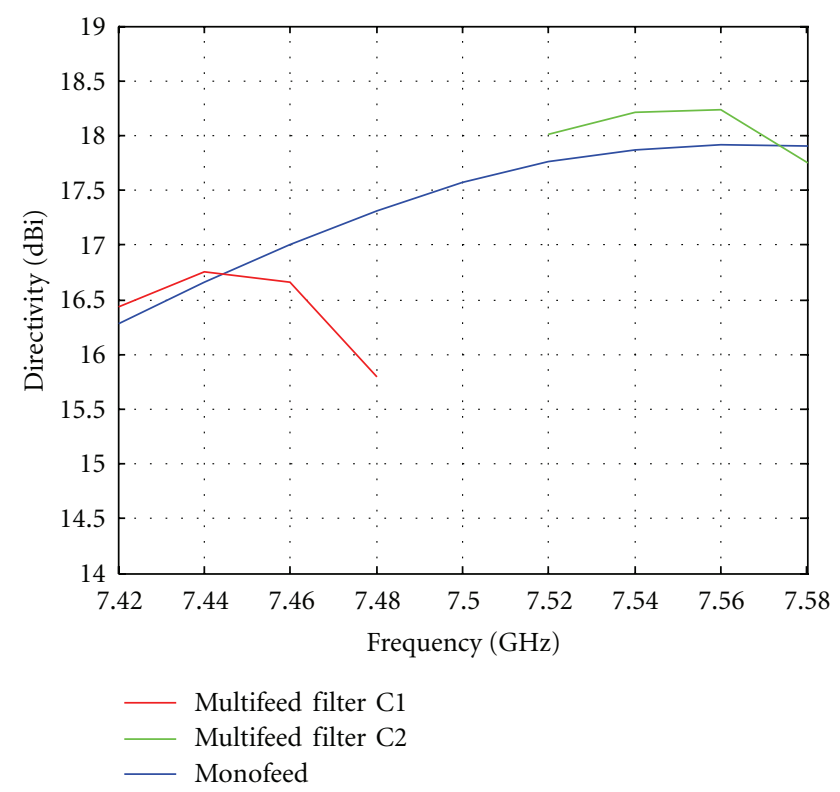

(b)

FIGURE 16: Directivity of the multifeed EBG antenna connected to filters inside both channels, compared to the ones of the monofeed antenna at $f_{L}(\mathrm{a})$ and $f_{H}(\mathrm{~b})$.

manufactured dual-band EBG antenna excited by one horn is given in Figure 4(b).

3.1.1. EBG Dual-Band Antenna Gain. In Figure 5, we present the measured gain of the fabricated monofeed antenna, compared to the simulated one. The maximum measured realized gain is around $15 \mathrm{dBi}$ and $16 \mathrm{dBi}$ at $5 \mathrm{GHz}\left(f_{L}\right)$ and $7.5 \mathrm{GHz}\left(f_{H}\right)$, respectively. The difference between the simulated and measurement gain is due to the difference between the simulated and measurement return loss and also to the precision error of the anechoic chamber, which is about $\pm 0.6 \mathrm{dBi}$.

3.1.2. Radiation Patterns. The radiation patterns in E-plane and H-plane are presented in Figure 6 for both frequency bands. We could observe a good agreement between the simulations and measurements. The half power beam widths of radiation patterns is about $12^{\circ}$ and $10^{\circ}$ at $f_{L}$ and $f_{H}$, respectively. Side lobes levels are about $-20 \mathrm{dBi}$ and $-25 \mathrm{dBi}$ for the lower and higher band, respectively. 


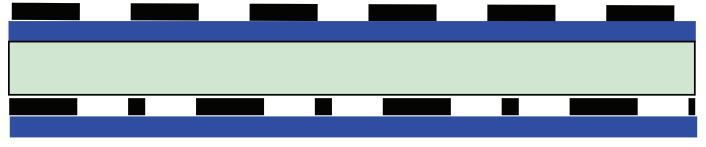

TABLE 1: Operating frequency channel.

\begin{tabular}{lll}
\hline & Lower band $(\mathrm{GHz})$ & Higher band $(\mathrm{GHz})$ \\
\hline Channel 1 (C1) & $4.95 \mathrm{GHz}-499 \mathrm{GHz}$ & $7.42 \mathrm{GHz}-7.49 \mathrm{GHz}$ \\
Channel 2 (C2) & $5.01 \mathrm{GHz}-5.05 \mathrm{GHz}$ & $7.51 \mathrm{GHz}-7.58 \mathrm{GHz}$ \\
\hline
\end{tabular}

given in Figure 9. We could observe a maximal directivity decrease of $2.5 \mathrm{dBi}$ and $1.2 \mathrm{dBi}$ at $f_{L}$ and $f_{H}$, respectively. This decrease is due to the coupling.

3.2.3. Radiation Patterns and E-Field Cartography. The radiation patterns for monofeed and multifeed EBG antenna for both frequency bands are given in Figure 10. As we can see, the radiation patterns for multifeed configuration are also influenced by the coupling between ports causing distortion in form of ripple in the main lobe of antenna radiation patterns.

Indeed, the coupling between ports induces a small FIGURE 17: Multifeed EBG antenna connected to filters by waveguide length.

For more details on the fabricated monofeed antenna and its measured results, please refer to [8].

3.2. Multifeed EBG Dual-Band Antenna. In this paragraph, we present the description of the multifeed antenna. It consists of using the same components as the monofeed one. However 7 feeding horns are used in this case. In order to have reflector antenna beams with an angular deviation equal to $0.56^{\circ}$ the feeds of the antenna must be separated by $70 \mathrm{~mm}$, (Figure 7). This value has been calculated for an offset reflector antenna with an $F / D$ ratio of 1.1 (focal length to diameter) and a $22^{\circ}$ half subtended angle. Due to the use of two symmetry planes in our simulations, only three horns are considered $(1,2$, and 6$)$. Initially, only port 1 is excited, whereas the others are loaded on the impedance waveguide. The polarization of ports 1 and 2 is orthogonal to the one of port 6.

Moreover, some changes are applied on both FSS in order to obtain the same directivity (18 dBi) and consequently the same reflector antenna illumination for the two frequency bands; see Figure 9.

3.2.1. S-Parameters. Figure 8 shows the S-parameters of the EBG multifeed antenna. The return loss is between $-5 \mathrm{dBi}$ and $-15 \mathrm{dBi}$ for the lower band $\left(f_{L}\right)$ and between $-8 \mathrm{dBi}$ and $-17 \mathrm{dBi}$ for the higher one $\left(f_{H}\right)$. The return loss can be improved by modifying the slot array properties (width and length of each slot). The coupling between ports, which have the same polarisation (ports 1 and 2), reaches a maximum of $-15 \mathrm{dBi}$ and $-22 \mathrm{dBi}$ at $f_{L}$ and $f_{H}$, respectively. However, the coupling between ports 1 and 6 is very low, since the polarizations of these ports are orthogonal to each other.

3.2.2. Directivity. The directivity of the multifeed EBG antenna compared to the one in monofeed configuration is As we can see, the E-field is less spread inside the cavity at $5.05 \mathrm{GHz}$ comparing to the case of the EBG antenna in monofeed configuration. This diminution explains the directivity decrease.

In order to solve this problem and isolate frequency as explained in the next section.

\section{Multifeed EBG Antenna Connected to Filters}

In this section, first we describe the principle of the multifeed EBG antenna which integrates filter devices. Then, we present the ideal filtering functions needed to reconstruct the radiation patterns. In the second part, we present the used filters and antenna performances when connected to filters.

4.1. Ideal Filtering Functions. As the multifeed EBG antenna is designed to operate simultaneously in two bands at 4.95$5.05 \mathrm{GHz}$ and $7.42-7.58 \mathrm{GHz}, 2$ dual-band filters, operating each inside a frequency channel of both bands (Table 1), are thus required to ensure the isolation between these channels. The principle of multifeed EBG antenna when connected to filters is shown in Figure 12.

Considering that port 1 operates at channel one $\mathrm{C} 1$ and port two operates at channel two $\mathrm{C} 2$, the radiation pattern of the structure when the port 1 is excited is given by [9]

$$
\Phi_{\text {Tot }}=\Phi_{1}+\Phi_{2} * \frac{S_{22-\text { filt-C2 }}(F 1) * S_{21 \text { Ant }}(F 1)}{1-S_{22-\text { filt-C2 }}(F 1) * S_{22 \text { Ant }}(F 1)},
$$

where $\Phi_{1}$ is the radiation pattern, obtained when port 1 is excited and port 2 is loaded by matched impedance and vice versa for $\Phi_{2}, S_{22-\text { filt-C2 }}(F 1)$ is the reflection coefficient of the filter operating in $\mathrm{C} 2, S_{21 \mathrm{Ant}}(F 1)$ is the coupling between two adjacent horns operating at the same polarization, and $S_{22 \mathrm{Ant}}(F 1)$ is the reflection coefficient of the EBG antenna.

In order to retrieve good radiation characteristics, the filters must manage the coupling in a constructive way. That radiating aperture of the EBG antenna as shown in Figure 11. channels, suited filters must be connected to antenna ports, 


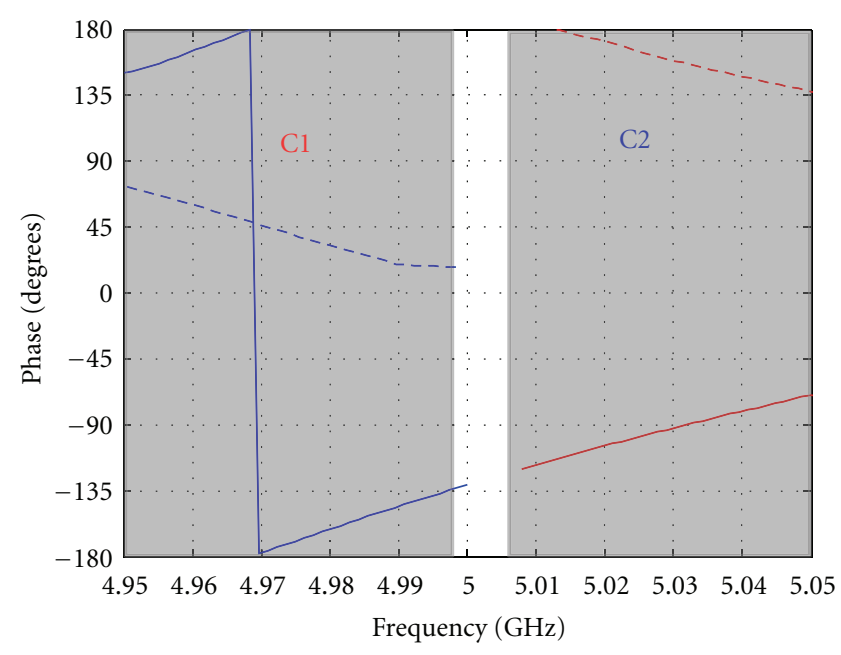

- Filter C1-ideal

- - Filter C1-Real

(a)

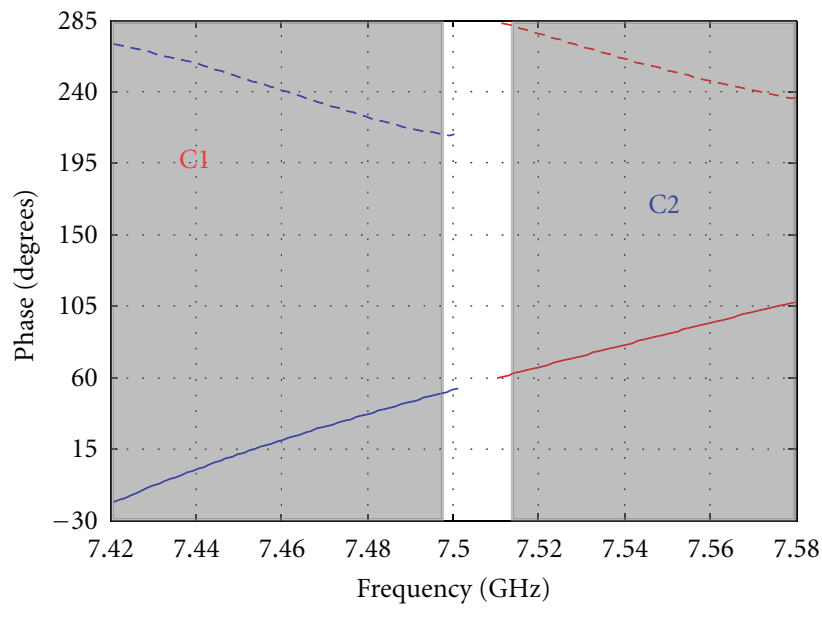

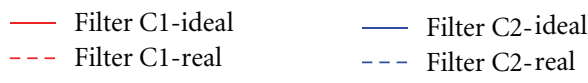

(b)

Figure 18: Out-of-band S22 phases profile of real filters, compared to the ones of ideal filters at $f_{L}(\mathrm{a})$ and $f_{H}(\mathrm{~b})$.

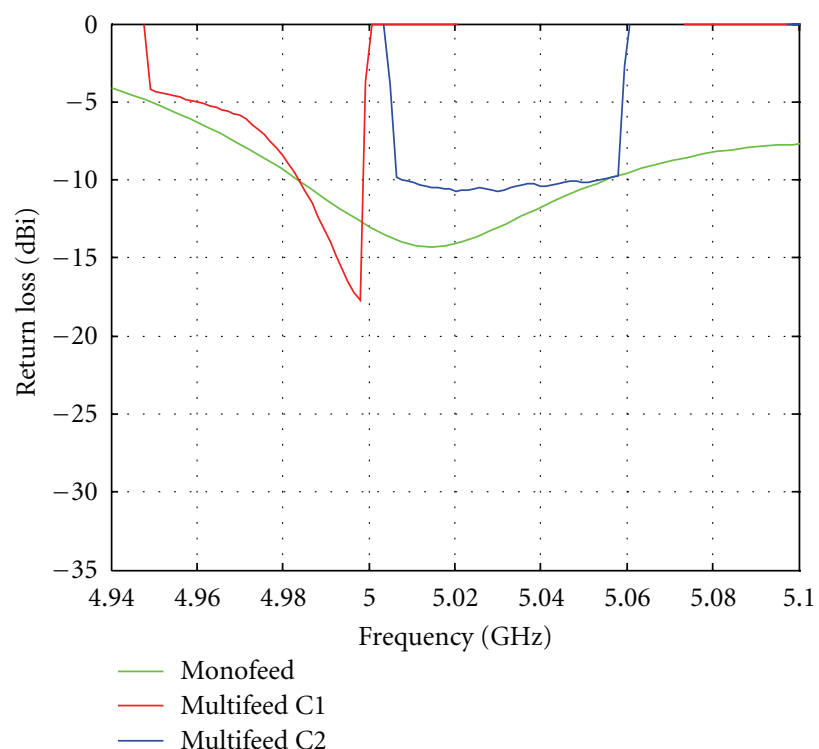

(a)

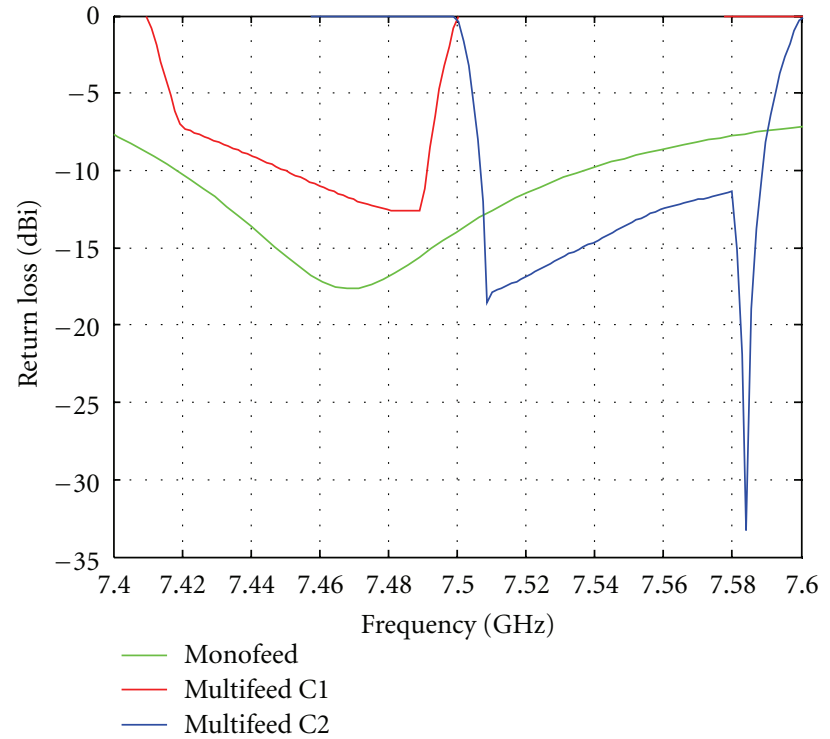

(b)

Figure 19: Return loss of the system inside both channels at $f_{L}$ (a) and $f_{H}$ (b).

is why each filter must act like a CCE with a specific phase profile outside its operating bands. The ideal out-of-band phase of each filter which guarantees good radiation patterns is given by [9]

$$
\operatorname{Arg}\left(S_{22-\text { filt-C2 }}(F 1)\right)=-\operatorname{Arg}\left(S_{21 \text { ant }}(F 1)\right),
$$

where $\operatorname{Arg}\left(S_{22-\text { filt-C2 }}(F 1)\right)$ is the reflection coefficient phase of the filter operating in $\mathrm{C} 2$ and $\operatorname{Arg}\left(S_{21 \text { Ant }}(F 1)\right)$ is the phase of the coupling between two adjacent horns operating at the same polarization.
In our case, the ideal out-of-band phase of each filter is presented in Figure 13, where we notice a rising slope of $140^{\circ}$ and $125^{\circ}$ at $f_{L}$ and $f_{H}$, respectively. However, it is impossible to realise matched filters with an out-of band rising slope phase, that is why filters with out of band falling slope could be used.

4.2. Used Filters. As previously explained the filter with an out-of band rising slope phase is considered impossible to achieve. Considering that filter $\mathrm{C} 1$ operates at channel one of each band and filter C2 operates at channel two of each 


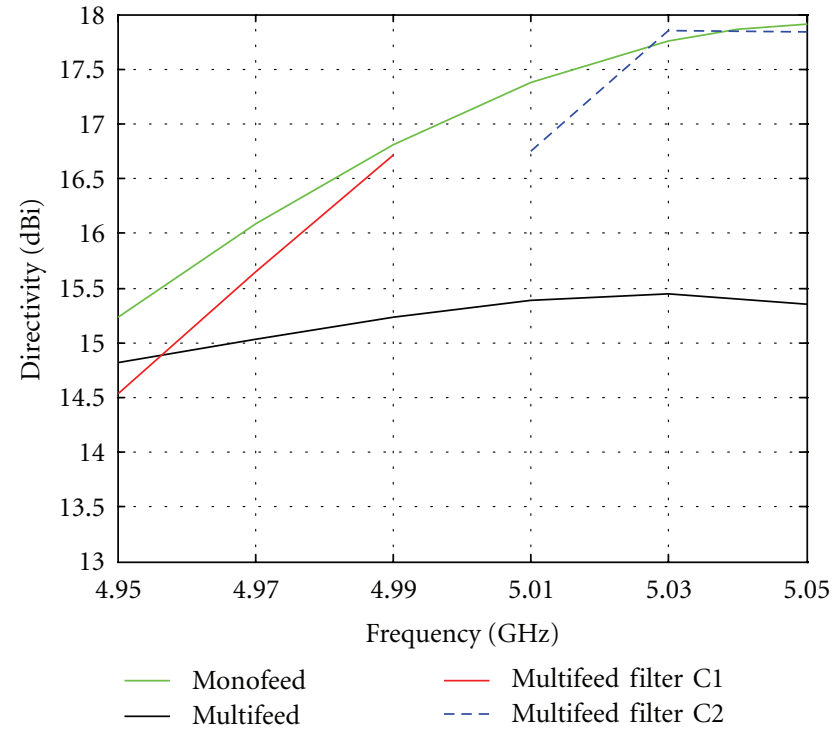

(a)

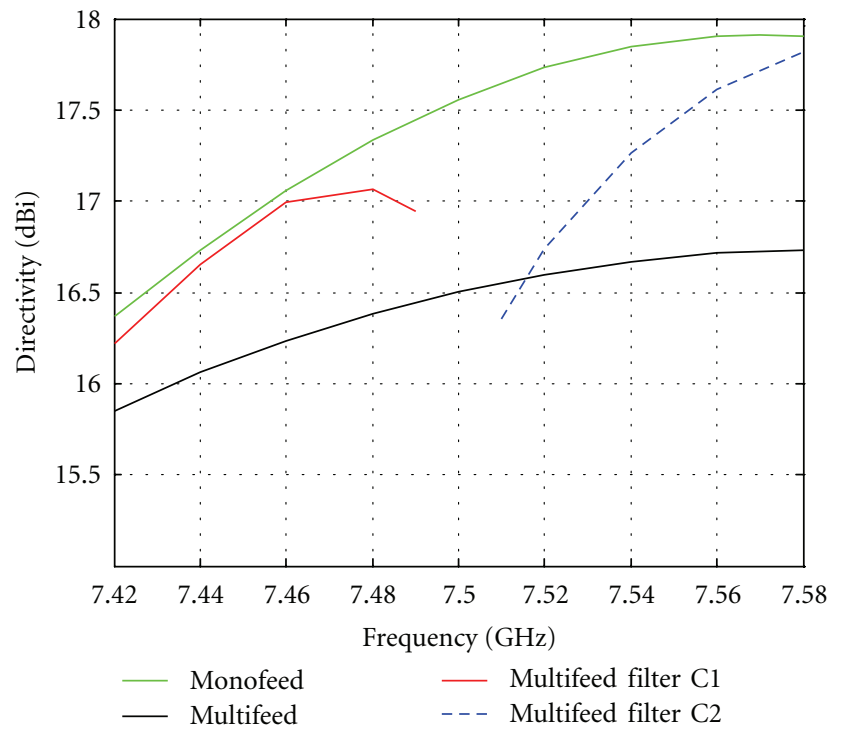

(b)

FIGURE 20: Directivity of the EBG dual-band antenna at $f_{L}$ (a) and $f_{H}(\mathrm{~b})$.

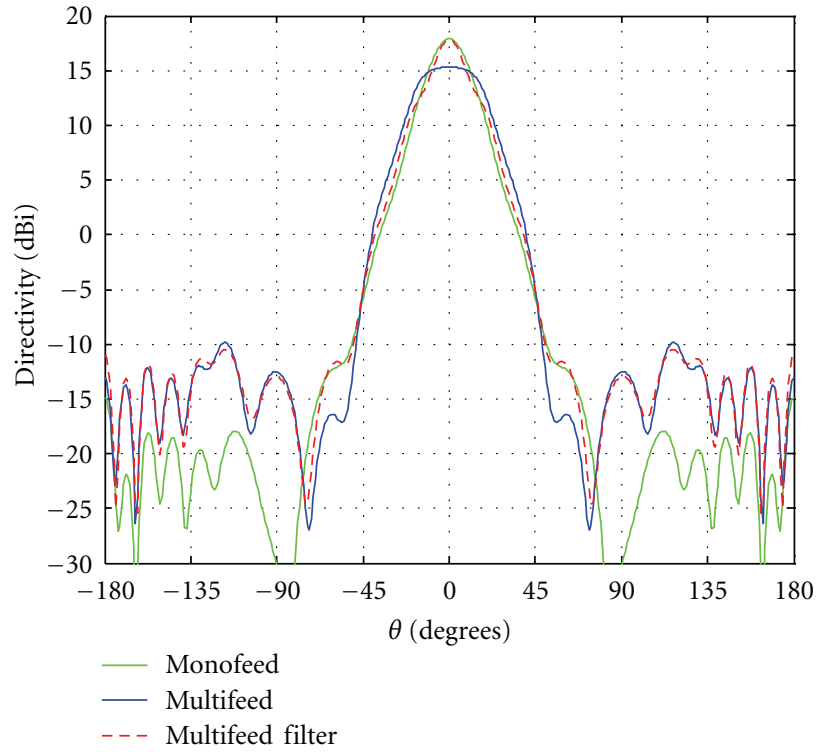

(a)

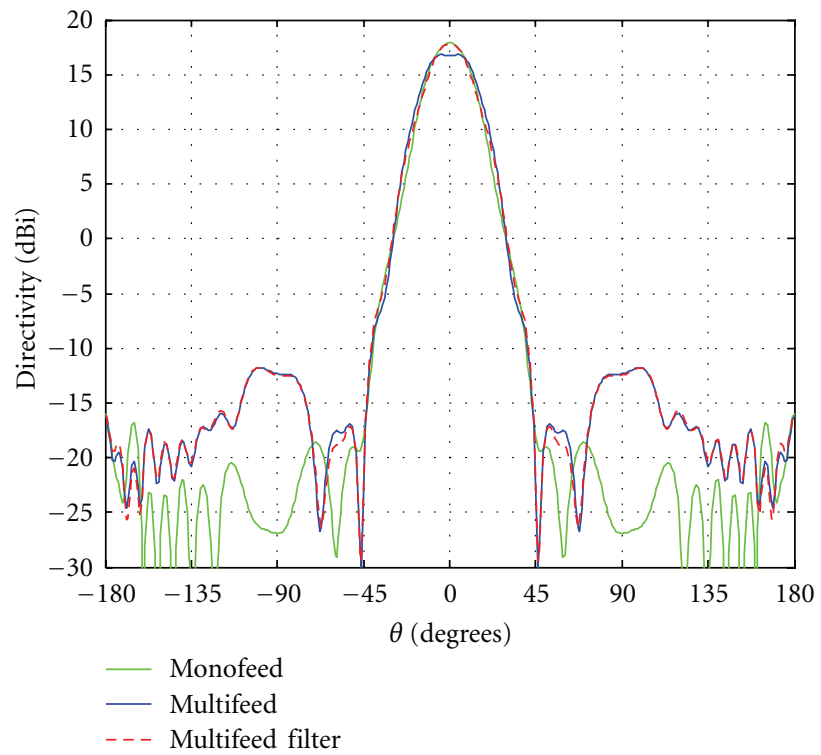

(b)

FIgURE 21: Radiation pattern of the EBG dual-band antenna at $f_{L}$ (a) and $f_{H}$ (b).

band, the used dual-band filter for each channel presents an out-of-band ideal reflection, Figure 14.

The out of band phase of the filter $\mathrm{C} 1$ has a negative slope of $50^{\circ}$ and $60^{\circ}$ at $f_{L}$ and $f_{H}$, respectively, while the one of the filter $\mathrm{C} 2$ is equal to $55^{\circ}$ at both frequency bands; they are compared to the ideal profiles in Figure 15. These phase profiles could be obtained by real filters [10].

Figure 16 shows the directivity of the multifeed EBG antenna loaded by used filters compared to the one of the antenna before connecting filters and the one of the monofeed antenna. As we can see, the use of the filters, which present an out-of-band downslope phase profile, does not improve the antenna performances, where we notice a maximal directivity decrease of $8 \mathrm{dBi}$ and $1.5 \mathrm{dBi}$ at $f_{L}$ and $f_{H}$, respectively. This is due to the fact that the phase values of the real filters are far from those of the ideal ones; in Figure 15, we can notice a maximum phase difference of $230^{\circ}$ and $150^{\circ}$ at $5.05 \mathrm{GHz}$ and $7.5 \mathrm{GHz}$, respectively.

In order to solve this problem, waveguide length phase shifters are added between the filters output and the horns input. As the same horn is used to excite the two frequency bands, the same waveguide length phase shifters must be used to shift the phase profile for both frequency bands. In this case, for each channel, one waveguide length phase 


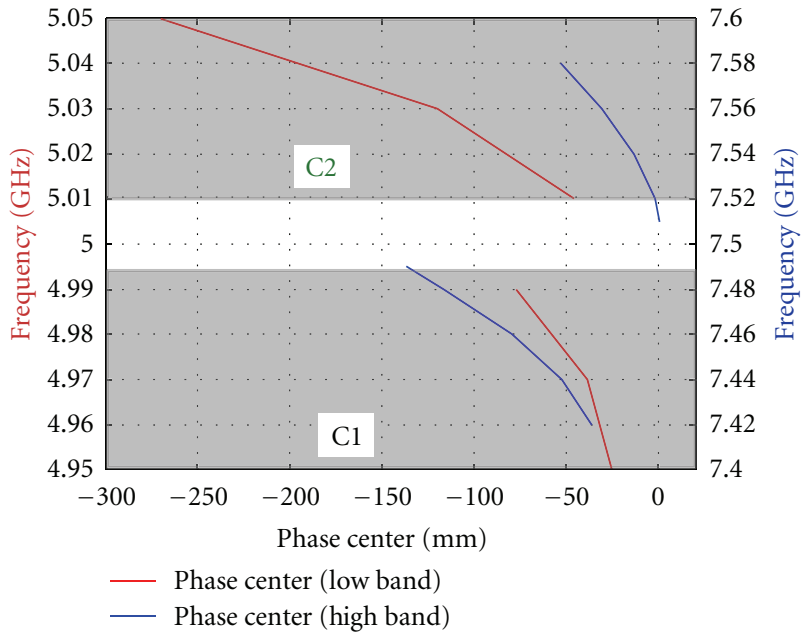

FIGURE 22: The phase center position for multifeed EBG dual-band antenna connected to used filters.

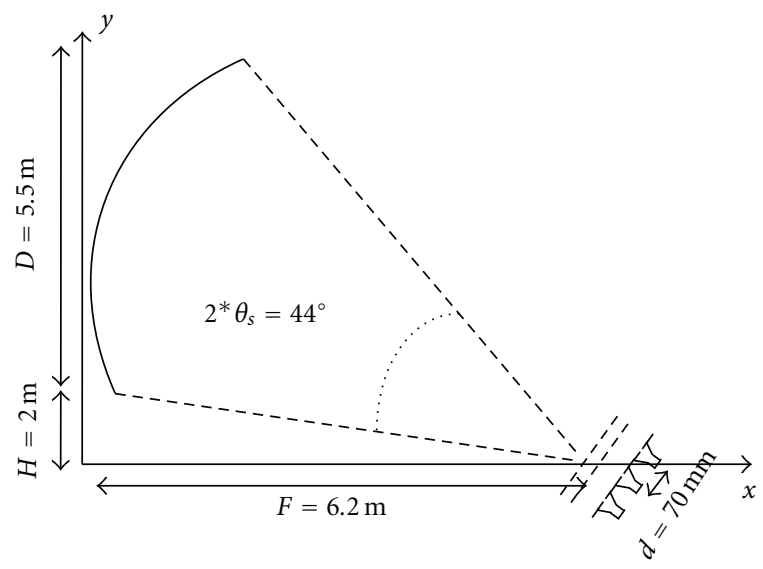

Figure 23: Reflector antenna geometry.

shifter must be used. As the phase difference is not the same for both band of each channel, a parameter sweep of the filter position has been performed in order to locate the optimal position that provides the best performance in terms of directivity for each channel. It has been found that the filter $\mathrm{C} 1$ should be placed $20 \mathrm{~mm}$ behind the port 1 in order to obtain the best performance at the channel 2. Likewise, the filter C2 should be placed $41 \mathrm{~mm}$ behind the port 2 in order to obtain the best performance at the channel 1, as shown in Figure 17.

The new phase position of the used filters compared to the one of ideal filters is given in Figure 18.

4.3. System Performances. The rectangular waveguides lengths are now added to the filter's output, which matches the rectangular input of the horns in order to extract system performances (antenna plus waveguide length phase shifters plus filters).

4.3.1. Return Loss. Figure 19 shows the reflection coefficient of the filter-feed combination. As can be noticed the latter is between $-5 \mathrm{dBi}$ and $-10 \mathrm{dBi}$ at the lower band and between $-7 \mathrm{dBi}$ and $-18 \mathrm{dBi}$ at the higher one in both channels.

4.3.2. Directivity. The directivity in both channels at the two frequency bands is presented in Figure 20, where the improvement, compared to the one of the multifeed structure before connecting the filters, can be noticed. It can also be seen that the directivity at the beginning of each channel is not as well reconstructed as for the end of each channel, which is due to the fact that the out-of-band phase of the filters is rolling away from the ideal one (see Figure 18).

4.3.3. Radiation Patterns. The reconstructed radiation patterns in E-plane are presented in Figure 21 for both frequency bands and compared to the ones before connecting the filters. The half power beam widths of radiation patterns is about $9^{\circ}$ and $11^{\circ}$ at $f_{L}$ and $f_{H}$, respectively. The side lobes level is always lower than $-15 \mathrm{dBi}$ from the maximum for both frequency bands.

4.3.4. Phase Center. As the dual-band EBG antenna should be used to feed a reflector antenna, the EBG antenna phase center must be identical at the two frequency bands. To define the location of the phase center precisely, several methods can be used [11]. The phase center is considered as the reference point that allows a minimum variation of the far field radiation phase within a defined solid angle $\theta_{S}$.

Figure 22 shows the phase center variation over both frequency bands (4.95-5.05) GHz and (7.45-7.6) GHz, where we notice a variation of $240 \mathrm{~mm}\left(4 \lambda_{0}\right)$ in the first band and about $140 \mathrm{~mm}\left(3.5 \lambda_{0}\right)$ in the second band behind the ground plan. This variation of the phase center position leads to several inconvenient like side lobes increasing and reflector efficiency decreasing.

\section{Coverage Characterization}

5.1. Reflector Description. In order to study the performances of the complete system (filter-feed-reflector) the EBG dualband antenna is placed at $110 \mathrm{~mm}$ in front of the reflector focal plane. This chosen value is the optimal one which minimises the effect of the phase center variation at both frequency bands in order to maximize the reflector antenna's performances. The studied offset reflector antenna is chosen with a $1.1 \mathrm{~F} / \mathrm{D}$ ratio in order to obtain multispot coverage without too many phase aberrations which can deteriorate its performances (Figure 23).

5.2. Feed Efficiency. The efficiency is an important parameter which characterizes the performances of a reflector antenna. In fact, a low efficiency requires more power at the output of the active part to realize good earth coverage. The efficiency of a reflector antenna can be written as a product of different efficiency terms. Each of them is a gain factor which represents a type of losses on the reflector antenna. This method, often used [12], is a good way to assess the importance of each factor and to isolate the parameters which affect the 


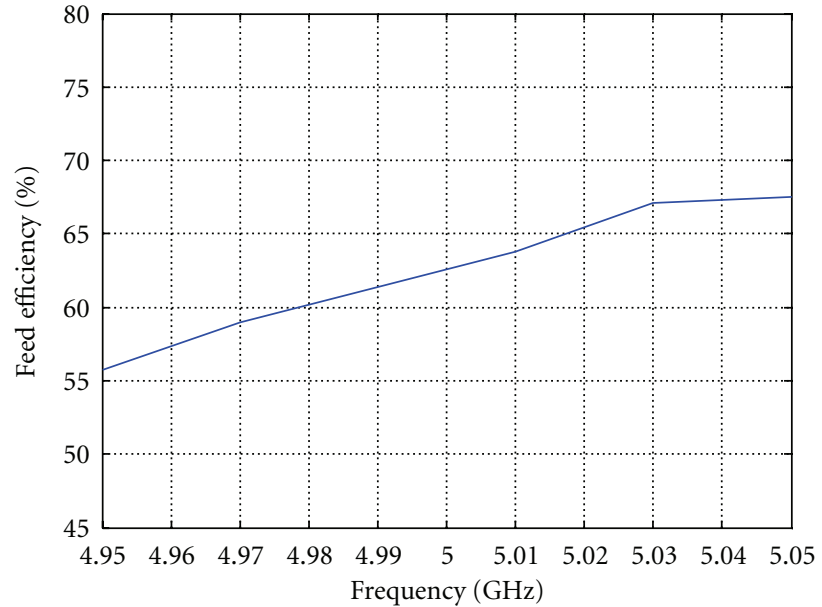

(a)

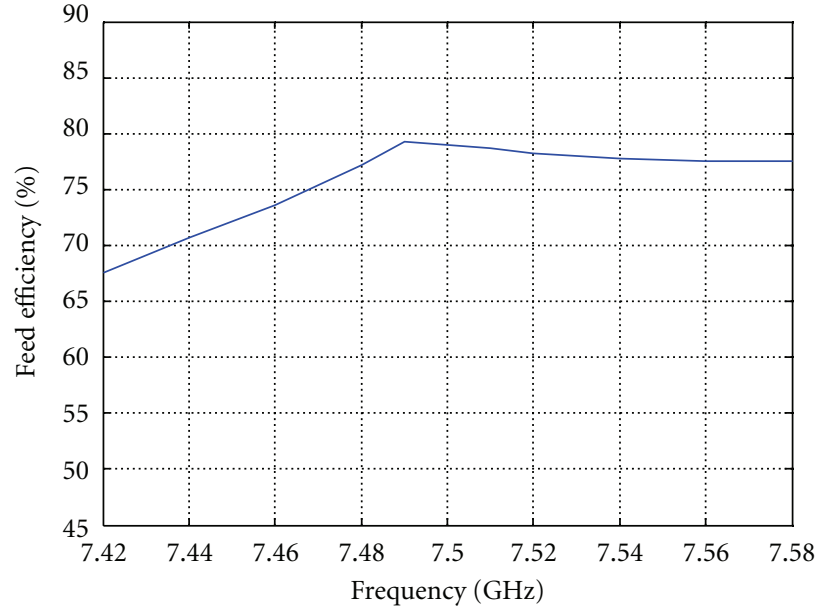

(b)

FIGURE 24: Feed efficiency comparison for the lower (a) and higher (b) frequency band.

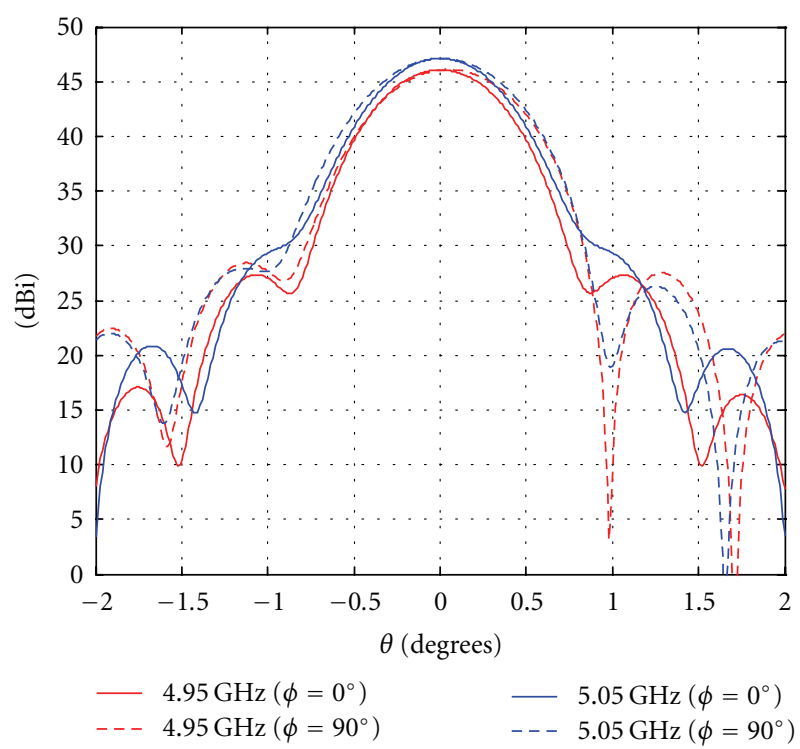

(a)

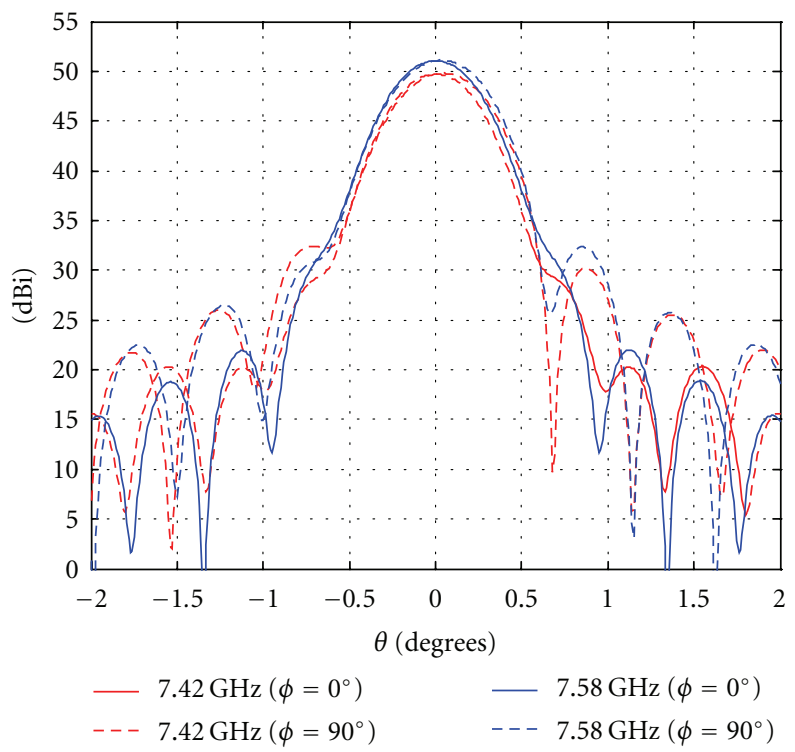

(b)

FIgURE 25: Reflector antenna radiation patterns with the EBG dual band at $f_{L}$ (a) and $f_{H}(\mathrm{~b})$.

total efficiency. The reflector antenna efficiency is calculated thanks to

$$
\eta=\eta_{s} \eta_{t} \eta_{\mathrm{pol}} \eta_{\mathrm{ph}}
$$

where $\eta_{s}, \eta_{t}, \eta_{\mathrm{pol}}$, and $\eta_{\mathrm{ph}}$ express, respectively, the spillover losses, feed taper losses, polarization losses, and the phase aberrations losses.

The evolution of reflector's efficiency versus frequency can be seen on Figure 24. As we can see, the efficiency is between $55 \%$ and $67 \%$ for the lower band and between $67 \%$ and $80 \%$ for the higher band. The difference between the efficiency for both frequency bands is due to the side lobes level which is higher at the lower band (see Figure 21).
5.3. Radiation Patterns. The radiation patterns of the reflector antenna illuminated by the nondefocused EBG dual-band antenna for both frequency bands are given in Figure 25. The half power beam widths of radiation patterns is about $0.7^{\circ}$ and $0.48^{\circ}$ at $f_{L}$ and $f_{H}$, respectively. The side lobes level is always lower than $-18 \mathrm{dBi}$ for both frequency bands. The cross-polarization level is always better than $-33 \mathrm{dBi}$ for the two frequency bands.

5.4. EOC Gain. The EOC gain for both bands and for all spots is presented in Figure 26. It is better than the $42 \mathrm{dBi}$ required in specifications at both channels except for few spots at the higher band. 


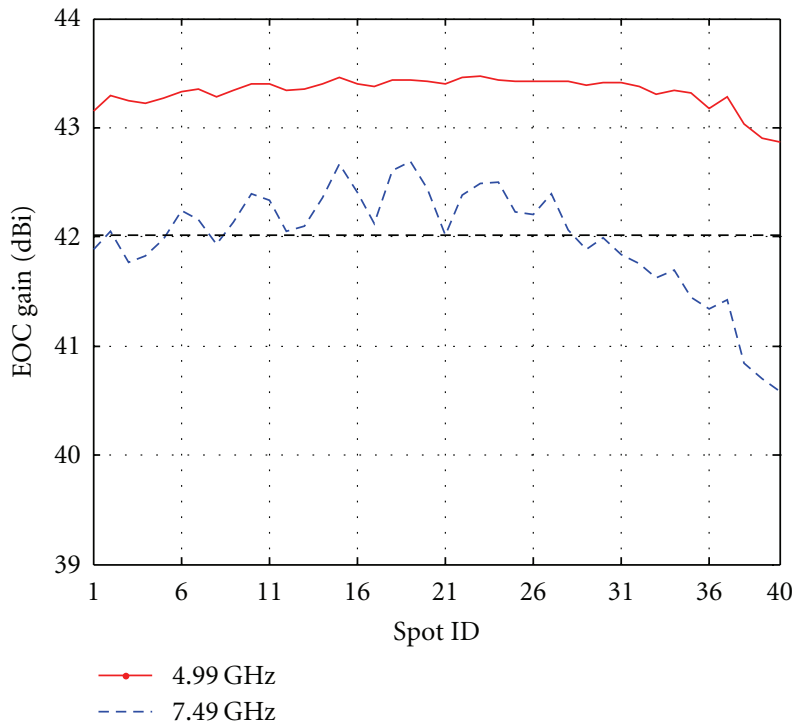

(a)

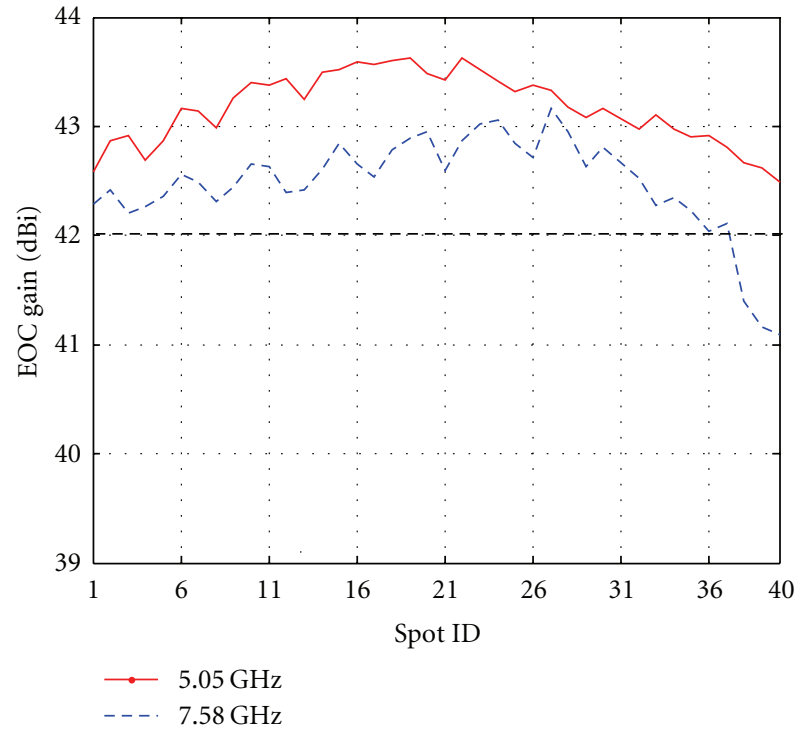

(b)

Figure 26: EOC gain on the coverage at channel 1 (a) and channel 2 (b).

\section{Conclusion}

In this paper, a multifeed EBG dual-band antenna used to feed a reflector antenna for spatial applications is proposed. The mutual coupling between feeds disturbs the radiation, thus the use of filters is required to reconstruct radiation characteristics and isolate channels. The multifeed EBG antenna presents the same phase center and the same main lobe for the two frequency bands, allowing it to feed a reflector antenna with a good efficiency. A filter-feed system with enhanced performance is then presented. This antenna is suitable for a reflector antenna with an $F / D$ ratio of 1.1 . The results of the offset reflector fed by the multifeed EBG dual-band antenna show a maximum efficiency of about $67 \%$ and for both frequency bands, respectively. A maximum EOC gain better $42 \mathrm{dBi}$ has been obtained for both frequency bands, except for few spots, where some changes could be made on the EBG antenna in order to improve performances. On the other hand, the slots array could be modified in order to improve the antenna return loss.

\section{References}

[1] D. Le Doan, E. Amyotte, C. Mok, and J. Uher, "AnikF2 Ka-band transmit multibeam antenna," in Proceedings of International Symposium on Antenna Technology and Applied Electromagnetics (ANTEM '04), July 2004.

[2] G. Caille, Y. Cailloce, B. Demolder, and G. Bekaert, "High gain multibeam antenna demonstrator for Ka band satellite," Alcatel Telecommunications Review, 2001.

[3] K. Lang, M. Eick, and D. Nakatani, "A 6-4 and 30-20 (or 1412) $\mathrm{GHz}$ dual foci offset paraboloidal reflector antenna," in Proceedings of the Antennas and Propagation Society International Symposium (APS '75), vol. 13, pp. 391-395, 1975.

[4] E. Amyotte, Y. Demers, L. Martins-Camelo et al., "High performance communications and tracking multi-beam antennas," in Proceedings of the European Conference on Antennas and Propagation (EuCAP '06), Nice, France, November 2006.

[5] R. Chantalat, C. Menudier, M. Thevenot, T. Monediere, E. Arnaud, and P. Dumon, "Enhanced EBG resonator antenna as feed of a reflector antenna in the Ka band," IEEE Antennas and Wireless Propagation Letters, vol. 7, pp. 349-353, 2008.

[6] C. Menudier, R. Chantalat, E. Arnaud, M. Thevenot, T. Monediere, and P. Dumon, "EBG focal feed improvements for Ka-band multibeam space applications," IEEE Antennas and Wireless Propagation Letters, vol. 8, pp. 611-615, 2009.

[7] A. Neto, N. Llombart, G. Gerini, M. Bonnedal, and P. De Maagt, "EBG enhanced feeds for high aperture efficiency reflector antennas," in Proceedings of the European Conference on Antennas and Propagation (EuCAP '06), Nice, France, November 2006.

[8] A. Kanso, R. Chantalat, M. Thevenot, E. Arnaud, and T. Monediere, "Offset parabolic reflector antenna fed by EBG dual-band focal feed for space application," IEEE Antennas and Wireless Propagation Letters, vol. 9, pp. 854-858, 2010.

[9] H. Chreim, R. Chantalat, M. Thèvenot et al., "Analysis of capabilities to achieve overlapped radiating apertures by using a multi-feed ebg structure loaded by passive filtering functions," in Proceedings of the 4th European Conference on Antennas and Propagation (EuCAP '10), Barcelona, Spain, April 2010.

[10] U. Naeem, Contributions au développement de sous systèmes filtre antennes avancés, Ph.D. thesis, University of Limoges, 2010.

[11] P. S. Kildal, "Combined E and $\mathrm{H}$ plane phase centers of antenna feeds," IEEE Transactions on Antennas and Propagation, vol. 31, no. 1, pp. 199-202, 1983.

[12] C. A. Balanis, Antenna Theory, Analysis and Design, chapter 13, John Wiley \& Sons, New York, NY, USA. 

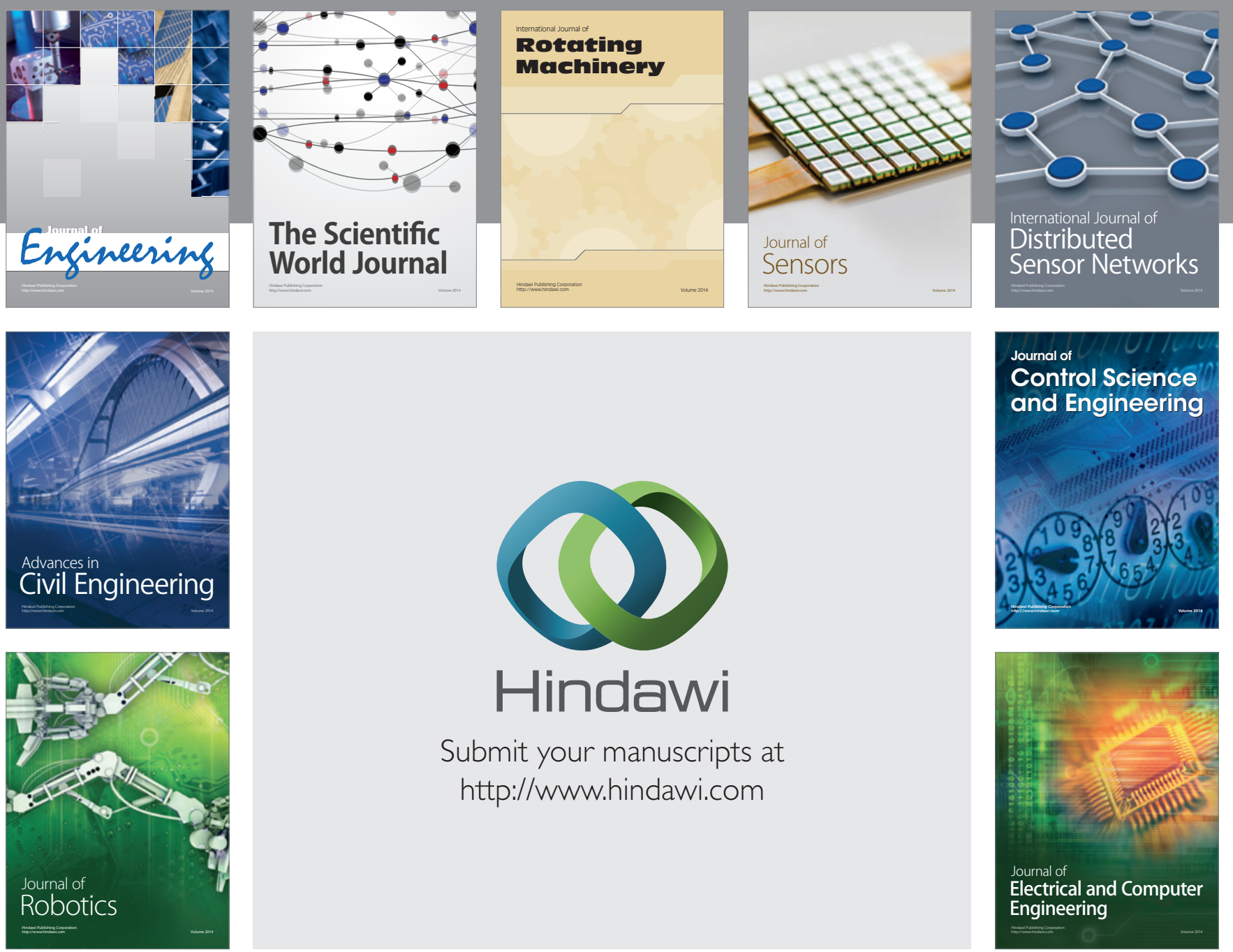

Submit your manuscripts at

http://www.hindawi.com
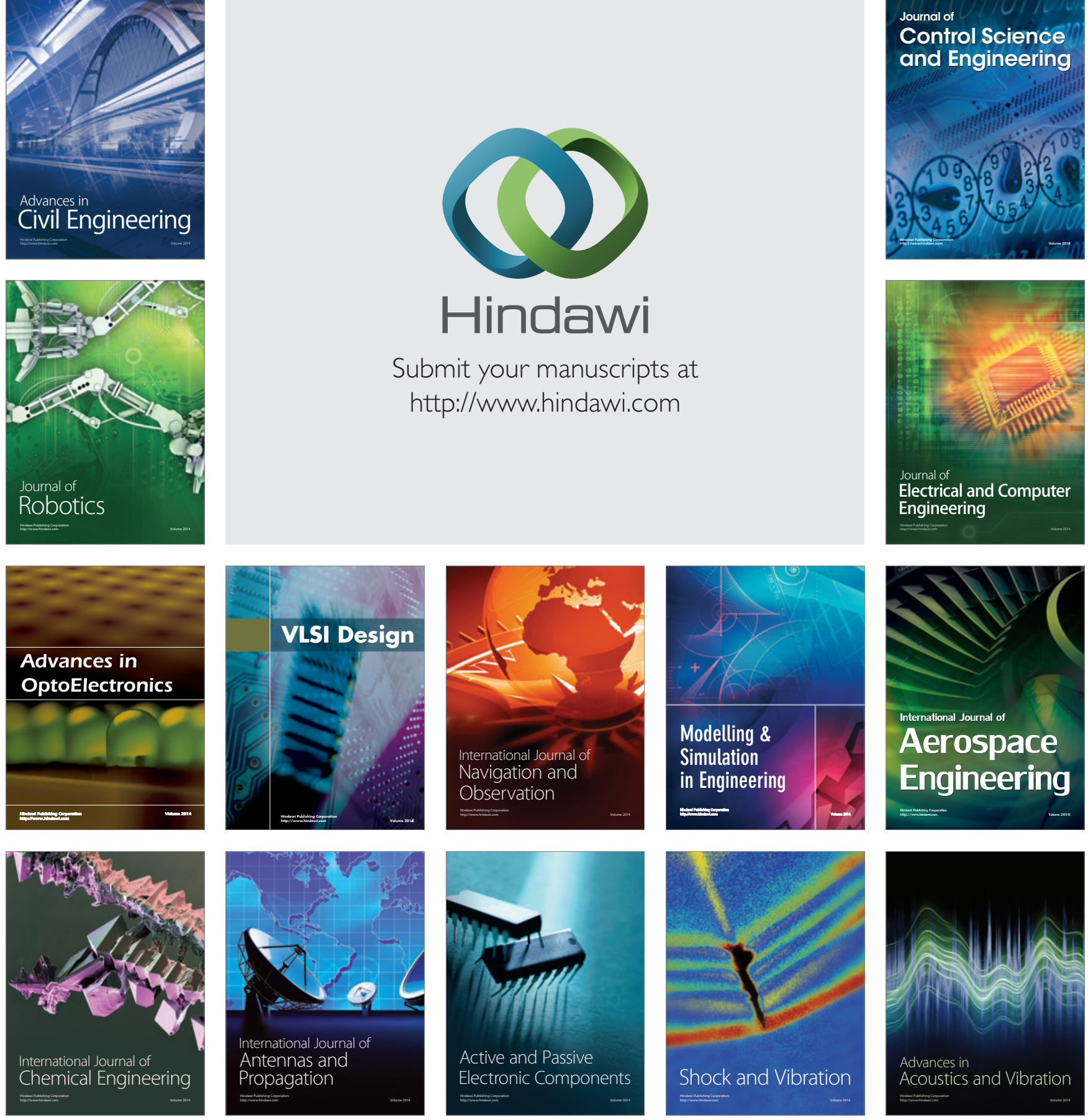